\title{
Chest computed tomography scan findings of coronavirus disease 2019 (COVID-19) patients: a comprehensive systematic review and meta-analysis
}

\author{
Mohammad Karimian ${ }^{1, A, B, C, D, E, E, G,}$, Milad Azami ${ }^{2}, A, B, C, D, E, E, G$ \\ 'Department of General Surgery, Faculty of Medicine, Ilam University of Medical Sciences, Ilam, Iran \\ ${ }^{2}$ Faculty of Medicine, Ilam University of Medical Sciences, Ilam, Iran
}

\section{Abstract}

Introduction: Numerous cases of pneumonia caused by coronavirus disease 2019 (COVID-19) were reported in Wuhan, China. Chest computed tomography (CT) scan is highly important in the diagnosis and follow-up of lung disease treatment. The present meta-analysis was performed to evaluate chest CT scan findings in COVID-19 patients.

Material and methods: All research steps were taken according to the Meta-Analysis of Observational Studies In Epidemiology (MOOSE) protocol and the final report was based on Preferred Reporting Items for Systematic Reviews and Meta-Analyses (PRISMA) guidelines. We registered this review at the International Prospective Register of Systematic Reviews (PROSPERO, CRD42019127858).

Results: Forty eligible studies including 4598 patients with COVID-19 were used for meta-analysis. The rate of positive chest CT scan in patients with COVID-19 was 94.5\% (95\% CI: 91.7-96.3). Bilateral lung involvement, pure ground-glass opacity (GGO), mixed (GGO pulse consolidation or reticular), consolidation, reticular, and presence of nodule findings in chest CT scan of COVID-19 pneumonia patients were respectively estimated to be $79.1 \%$ (95\% CI: 70.8-85.5), 64.9\% (95\% CI: 54.1-74.4), 49.2\% (95\% CI: 35.7-62.8), 30.3\% (95\% CI: 19.6-43.6), 17.0\% (95\% CI: 3.9-50.9) and 16.6\% (95\% CI: 13.6-20.2). The distribution of lung lesions in patients with COVID-19 pneumonia was peripheral (70.0\% [95\% CI: 57.8-79.9]), central (3.9\% [95\% CI: 1.4-10.6]), and peripheral and central (31.1\% [95\% CI: 19.5-45.8]). The pulmonary lobes most commonly involved were the right lower lobe (86.5\% [95\% CI: 57.7-96.8]) and left lower lobe (81.0\% [95\% CI: 50.5-94.7]).

Conclusions: The most important outcomes in chest CT scan of patients with COVID-19 pneumonia were bilateral lung involvement, GGO or mixed (GGO pulse consolidation or reticular) patterns, thickened interlobular septa, vascular enlargement, air bronchogram sign, peripheral distribution, and left and right lower lobes involvement. Our study showed that chest CT scan has high sensitivity in the diagnosis of COVID-19, and may therefore serve as a standard method for diagnosis of COVID-19.

Key words: computed tomography, COVID-19, meta-analysis.

\section{Introduction}

In December 2019, numerous cases of pneumonia of unknown cause were reported in Wuhan, Hubei Province, China. On January 7, 2020, a novel coronavirus, severe acute respiratory syndrome coronavirus-2 (SARS-CoV-2), was identified as the causative organism by Chinese experts by performing real-time polymerase chain reaction (real-time PCR) on patients' respiratory tract specimens.
It was subsequently named 2019-nCoV by the World Health Organization (WHO) [1]. There is also evidence that it can be transmitted through respiratory droplets and contact with infected patients as well as fecal-oral transmission [2,3]. Etiologically speaking, virulence of a pathogen may increase sharply during host shifts [4,5], and in contrast, the virulence may decrease through prolonged host-parasite interactions [6].

\section{Correspondence address:}

Dr. Milad Azami, Faculty of Medicine, Ilam University of Medical Sciences, Ilam, Iran, e-mail: milad98azami@gmail.com

Authors' contribution:

A Study design · B Data collection · C Statistical analysis · D Data interpretation · E Manuscript preparation · F Literature search · G Funds collection 
Coronavirus disease 2019 (COVID-19) is primarily transmitted through respiratory droplets and close contact, and the incubation period is usually between 1 and 14 days. The common symptoms include fever, dry cough, fatigue, and the gradual onset of shortness of breath. People who carry the virus are the source of infection, even during the incubation period. Early detection of the disease or the virus carrier is the key to prevent further spread. However, confirmation of the infection requires a nucleic acid detection kit. The virus can be identified in swabs, secretions, and sputum from the respiratory tract, blood, or feces [7].

Computed tomography (CT) scan is highly important in the diagnosis and follow-up of lung disease treatment. In a review of different studies, one may find that the imaging features of COVID-19 pneumonia are varied, from their natural appearance to diffuse changes in the lungs. In addition, different radiological patterns are observed at different times over the course of the disease. Since the onset of symptoms and acute respiratory distress syndrome (ARDS) was short-lived in the first cases of COVID-19 pneumonia, early detection of the disease is essential for the management of these patients [8].

Numerous studies have been performed regarding the findings of CT scans in COVID-19 patients and the results are inconsistent $[3,8-46]$. According to previous studies, lesions in patients with COVID-19 show ground-glass opacity (GGO) or mixed (GGO pulse consolidation or reticular) patterns, and are likely to have peripheral distribution, bilateral involvement, lower lobe dominance, and multilobe distribution [8-15]. In a systematic review and metaanalysis, a structured review of all documentation and their composition can provide a more comprehensive picture of all dimensions of the subject. One of the main goals of meta-analysis, which is a combination of different studies, is to reduce the differences between parameters by increasing the number of studies involved in the analysis process. Another noteworthy goal of meta-analysis is to find inconsistencies between the results and their causes [47-49]. The present meta-analysis was conducted to evaluate CT scan findings of COVID-19 at the time of admission.

\section{Material and methods}

\section{Study protocol}

The International Prospective Register of Systematic Reviews (PROSPERO) database and international databases were first reviewed to find relevant published or ongoing projects. All research steps were taken according to the Meta-Analysis of Observational Studies in Epidemiology (MOOSE) protocol [49] and the final report was based on the Preferred Reporting Items for Systematic Reviews and Meta-Analyses (PRISMA) guideline (Suppl Table 1) [50]. Each stage of the study was conducted by two independent authors. Disagreements were resolved by discussion or a third author was involved. We registered this review at PROSPERO (registration number: CRD42020178078) (Suppl Figure 1). Available at: https://www.crd.york.ac.uk/ prospero/display_record.php?RecordID $=178078$.

\section{Literature search}

We searched the Web of Science: ISI, Medline-Ovid, Science Direct, Scopus, EMBASE, PubMed/Medline, Cochrane Library (Cochrane Database of Systematic Reviews - CDSR), EBSCO, CINAHL and Google Scholar databases using the following keywords: "2019 nCoV", "Novel coronavirus", "COVID-19", "Novel coronavirus 2019", "Wuhan pneumonia", "Wuhan coronavirus", "acute respiratory infection", "COVID-19”, and "SARS-CoV-2", "CT scan", "Computed tomography”, "Radiology", "Radiography”, "Clinical Characteristics", "clinical features”, and "COVID-19". An example of a combined search within PubMed is as follows: ("2019 nCoV", OR "Novel coronavirus”, OR “COVID-19”, OR “Novel coronavirus 2019”, OR "Wuhan pneumonia”, OR "Wuhan coronavirus", OR "acute respiratory infection", OR "COVID-19”, OR “SARSCoV-2") AND ("CT scan" OR "Computed tomography" OR "Radiology” OR "Radiography” OR "Clinical characteristics” OR “clinical features” OR “COVID-19”).

The search was conducted on March 20, 2020. Additional studies were identified by reviewing the reference lists of relevant articles. No language restrictions were applied. Since the present study was based on a regular review of previous studies, approval of the organizational review board and patient satisfaction were not necessary. The research received no specific funding. Grey literature was found at medrxiv (https://www.medrxiv.org/) and manual search of related articles was also conducted.

\section{Inclusion and exclusion criteria}

Inclusion criteria were all cross-sectional epidemiological studies aimed at examining chest CT scan findings in COVID-19 patients from January 1, 2020 until March 20, 2020 without language restrictions. Exclusion criteria were as follows: 1 . Non-random sampling; 2 . Duplicate studies; 3. Studies on non-adult population (more than $10 \%$ of sample size being children); 4 . Being irrelevant; 5. Sample size smaller than 10 participants; 6. Diagnostic intervention for COVID-19 other than laboratory confirmation; 7. CT scan findings have not been verified by at least one radiology expert; 8 . Poor quality in qualitative evaluation; and 9. Case reports, review articles, and letters to the editor without quantitative data.

\section{Study selection and data extraction}

Two authors independently presented the results of the initial search with the title and abstract. At this stage, duplicate and unrelated studies were excluded. Duplicate 
studies were identified manually or using EndNote X9. Both authors then reviewed the full text of appropriate articles for the inclusion and exclusion criteria. Finally, the authors extracted the data independently from the articles. Any discrepancy between the data extractors was resolved by consensus or by a third author. It should be noted that when an article reported duplicate information from the same patients, both reports were combined to obtain the most complete data, but it was considered as one case.

The data summary form includes the following items: first author's name and year of publication, country and province, article references, study design, mean age and standard deviation, average duration from onset of symptoms until admission, time of performing CT scan, COVID-19 detection method, patient description, sample (respiratory secretions, blood, etc.), sample location (nasal, pharyngeal, etc.), number of patients (total, male and female), number of patients referred to the intensive care unit (ICU), quality of articles, outcomes information: positive chest CT scan in COVID-19 patients, including the sensitivity of chest CT, bilateral pneumonia, predominant chest CT scan patterns [GGO, mixed (GGO pulse consolidation or reticular), consolidation, reticular, and presence of nodule], other chest CT scan features (thickened interlobular septa, vascular enlargement, air bronchogram sign, bronchial wall thickening, bronchiolectasis, fibrous stripes, crazy-paving pattern, thickening of the adjacent pleura, pleural effusion and lymphadenopathy), distribution of lesions (peripheral, central, and peripheral pulse central), lobes involvement (right upper lobe, right middle lobe, right lower lobe, left upper lobe, and left lower lobe) and number of involved lobes (one lobe, two lobes, three lobes, four lobes, and five lobes).

\section{Qualitative evaluation}

Based on the type of studies, the adapted Newcastle-Ottawa Scale (NOS) was used to evaluate the risk of bias [51]. Three categories were defined: studies with scores less than 6 were low-quality studies, studies with scores of 6 or 7 were medium-quality, and studies with scores of 8 or 9 were highquality studies.

\section{Statistical analysis}

The $I^{2}$ index (with values ranging from 0 to $100 \%$ ) was used to evaluate the heterogeneity between studies; values above $75 \%$ indicate high heterogeneity, values within $50-74 \%$ indicate significant heterogeneity, values within $25-49 \%$ indicate moderate heterogeneity, and values below 25\% indicate low heterogeneity [52,53]. In addition, $p<0.1$ was also defined for heterogeneity. Meta-analysis was performed with at least three studies. In the case of low heterogeneity the fixed effects model, and otherwise the random effects model, was used to combine the studies. Results were reported as pooled prevalence and 95\% confidence interval. To identify the cause of heterogeneity, we could not perform subgroup analysis or meta-regression due to limitations. Sensitivity analysis for meta-analyses with at least five studies was performed by omitting one study at a time to evaluate the consistency of the results. Funnel plots and the Begg and Egger tests were used to evaluate publication bias. All analyses were performed using comprehensive meta-analysis. $P$-values less than 0.05 were considered statistically significant.

\section{Results}

\section{Description of included studies}

We identified 2266 potential articles from databases. After removing duplicates, there were 766 articles left. After evaluating the titles and abstracts, 76 articles were removed for at least one of the following reasons: non-random sampling $(n=10)$, studies on non-adult population $(n=3)$, being irrelevant $(n=650)$, diagnostic intervention for COVID-19 other than laboratory confirmation $(n=3)$, CT scans findings not verified by at least one radiology expert $(n=2)$, poor quality in qualitative evaluation $(n=0)$, and case reports, review articles, and letters to the editor without quantitative data $(n=58)$. Finally, 40 eligible studies with 4598 patients with COVID-19 were used for meta-analysis. This process is illustrated in Figure 1. All studies were conducted in China and all of them were of good or moderate quality and the details of studies are summarized in Table 1 . The mean age of the study participants was 50.52 years (95\% CI: 50.87-52.17).

\section{Risk of bias}

The risk of bias based on the NOS tool is shown in Table 1. Fourteen and 26 studies had moderate and good quality, respectively.

\section{Sensitivity of chest CT findings for COVID-19}

The sensitivity of chest CT scan in patients with COVID-19 was 94.5\% (95\% CI: 91.7-96.3; $I^{2}=98.6 \%$ ). The lowest and highest estimates were for studies by Haiyan et al. (61.5\%) and many other studies (100\%), respectively (Figure 1A).

\section{Bilateral lung involvement}

Bilateral lung involvement in chest CT scan with COVID-19 pneumonia was observed in $79.1 \%$ of patients $(95 \% \mathrm{CI}$ : 70.8-85.5) (Figure 2B).

\section{Predominant chest CT scan patterns}

Pure GGO, mixed (GGO pulse consolidation or reticular), consolidation, reticular, and presence of nodule findings in chest CT scan of COVID-19 pneumonia patients were observed in $64.9 \%$ (95\% CI: 54.1-74.4), 49.2\% (95\% CI: 


\begin{tabular}{|c|c|c|c|c|c|c|c|c|c|c|c|c|c|}
\hline \multicolumn{2}{|l|}{ 홀 } & \multirow{2}{*}{$\bar{\nu}$} & \multirow{2}{*}{$\begin{array}{l}a \\
F\end{array}$} & \multirow{2}{*}{$\begin{array}{l}a \\
2\end{array}$} & \multirow{2}{*}{$\begin{array}{l}\sim \\
\approx\end{array}$} & \multirow{2}{*}{\begin{tabular}{|l|} 
\\
$F$ \\
\end{tabular}} & \multirow{2}{*}{$\begin{array}{l}\infty \\
\infty\end{array}$} & \multirow{2}{*}{$\begin{array}{l}a \\
\stackrel{\infty}{=}\end{array}$} & \multirow{2}{*}{$\begin{array}{l}\infty \\
\infty\end{array}$} & \multirow{2}{*}{$\begin{array}{l}\infty \\
6\end{array}$} & \multirow{2}{*}{$\begin{array}{l}a \\
\bar{n}\end{array}$} & \multirow{2}{*}{$\begin{array}{l}a \\
\sigma\end{array}$} & \multirow{2}{*}{$\begin{array}{l}\infty \\
\wp\end{array}$} \\
\hline \multirow{3}{*}{$\frac{\frac{\tilde{N}}{\bar{y}}}{\frac{0}{\bar{c}}}$} & $\stackrel{I}{E}$ & & & & & & & & & & & & \\
\hline & 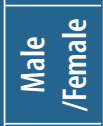 & $\stackrel{I}{I}$ & $\underset{\bar{D}}{\bar{\Sigma}}$ & $\underset{\widetilde{\sigma}}{\widehat{\sigma}}$ & $\underset{\infty}{\infty}$ & 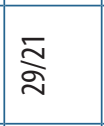 & 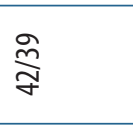 & $\stackrel{0}{\stackrel{0}{n}}$ & 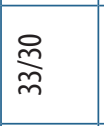 & 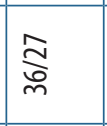 & 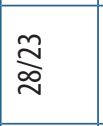 & 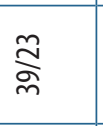 & 筞 \\
\hline & $\equiv$ & $\bar{\sim}$ & $\bar{\sigma}$ & 8 & gे & in & $\bar{\infty}$ & $\stackrel{\infty}{=}$ & ஜ & $\widetilde{\sigma}$ & $\bar{n}$ & $\widetilde{\sigma}$ & $\bar{\varrho}$ \\
\hline \multicolumn{2}{|l|}{ 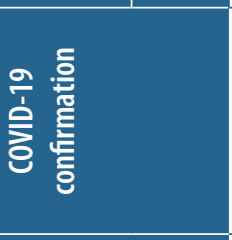 } & 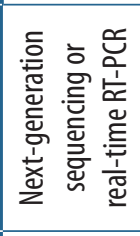 & 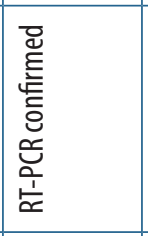 & 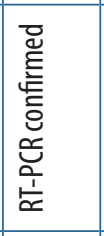 & 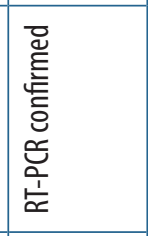 & 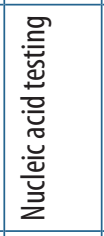 & 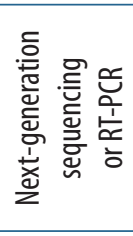 & 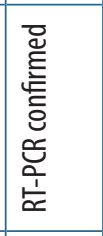 & 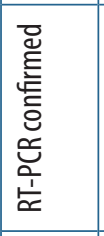 & 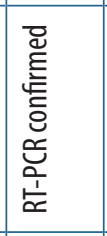 & 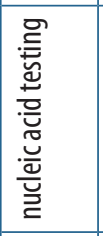 & 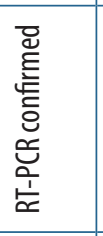 & 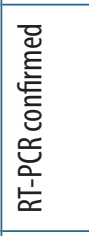 \\
\hline \multicolumn{2}{|l|}{ 亳 } & 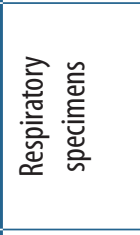 & 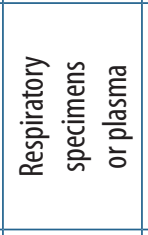 & 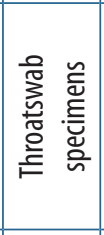 & 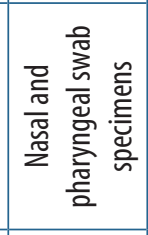 & 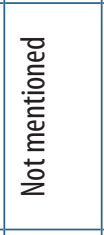 & 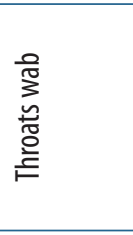 & 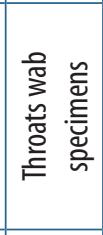 & 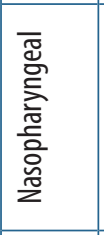 & 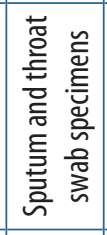 & 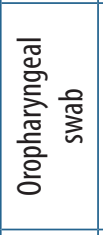 & 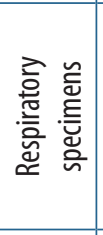 & 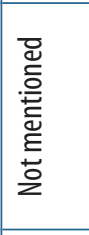 \\
\hline \multicolumn{2}{|l|}{ 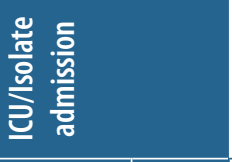 } & & $\stackrel{\infty}{m}$ & & $\frac{g}{\partial}$ & & & 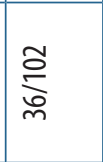 & & & & & \\
\hline \multirow{2}{*}{ 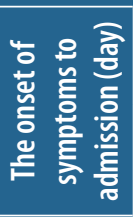 } & 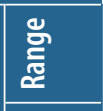 & & $\stackrel{\infty}{+}$ & & in & & & $\stackrel{\infty}{+}$ & & $\stackrel{+}{I}$ & & & \\
\hline & 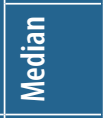 & & $\stackrel{\circ}{i}$ & & $\underset{0}{\infty}$ & & & $\stackrel{\circ}{i}$ & & $\stackrel{\stackrel{i}{i}}{ }$ & & & \\
\hline \multirow{2}{*}{ 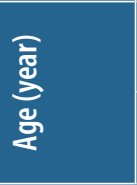 } & ทิ & 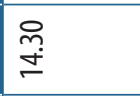 & $\begin{array}{l}\infty \\
\stackrel{\infty}{\sigma} \\
\stackrel{\sigma}{\sigma}\end{array}$ & $\bar{m}$ & $\stackrel{\stackrel{n}{n}}{m}$ & $\stackrel{\infty}{\circ}$ & $\stackrel{\circ}{\rightleftarrows}$ & & $\stackrel{2}{\check{n}}$ & $\begin{array}{l}\stackrel{\sim}{\hat{N}} \\
\stackrel{\sim}{\sim}\end{array}$ & $=$ & $\underset{\beth}{\beth}$ & $\stackrel{m}{\simeq}$ \\
\hline & 恋 & nి & $\stackrel{\circ}{\circ}$ & 瓷 & E & $\stackrel{\circ}{\ddot{q}}$ & $\tilde{q}$ & & 守 & $\stackrel{\circ}{\stackrel{9}{\sigma}}$ & 吕 & 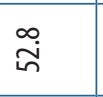 & $\begin{array}{l}\text { J } \\
\forall \\
\end{array}$ \\
\hline \multicolumn{2}{|l|}{$\begin{array}{l}\text { 흘 } \\
\text { 言 } \\
\text { 홍 }\end{array}$} & 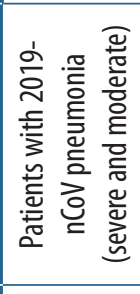 & 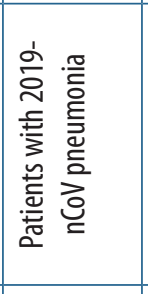 & 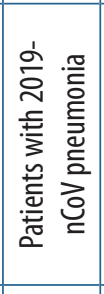 & 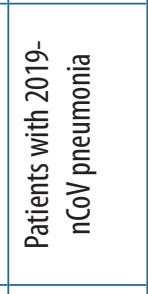 & 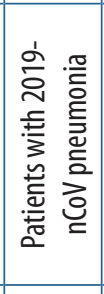 & 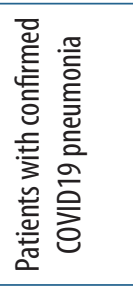 & 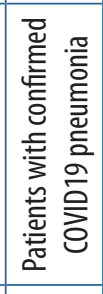 & 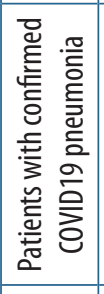 & 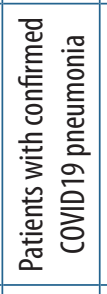 & 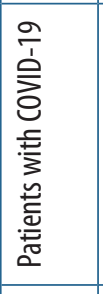 & 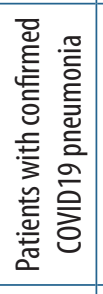 & 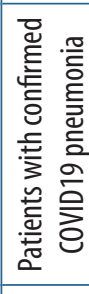 \\
\hline \multicolumn{2}{|l|}{ 言 } & 疍胥 & 兽蛋 & 胥禀 & 胥 & 突 莺 & 兽胥 & 窝䲶 & 胥 & $\mid$ & 总爱 & 茑胥 & 预 空 \\
\hline \multicolumn{2}{|l|}{ 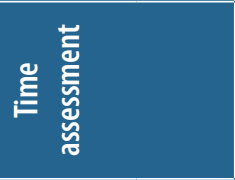 } & 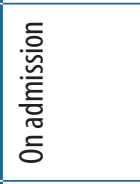 & 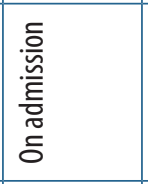 & 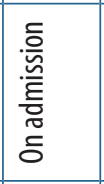 & 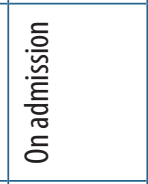 & 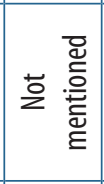 & 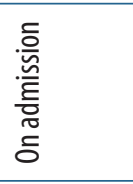 & $\begin{array}{l}\text { 흔 } \\
\text { 产 } \\
\overline{0} \\
\bar{\delta}\end{array}$ & 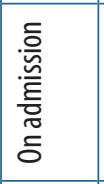 & 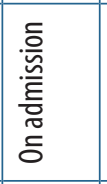 & 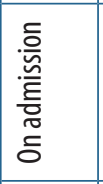 & 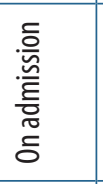 & 할 \\
\hline \multicolumn{2}{|l|}{ ֻัँ } & 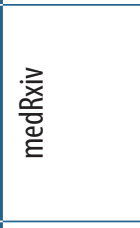 & 壱 & 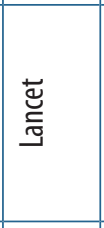 & 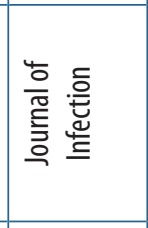 & 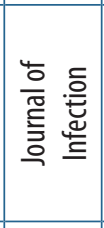 & 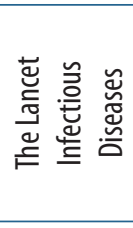 & $\sum_{i}^{\frac{\pi}{x}}$ & 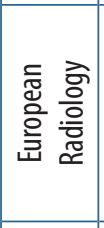 & 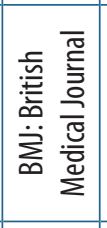 & 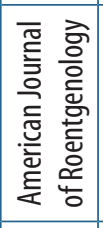 & 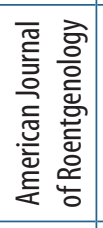 & 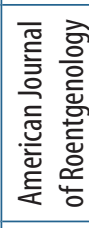 \\
\hline \multicolumn{2}{|c|}{ 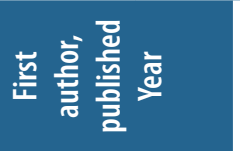 } & जั & 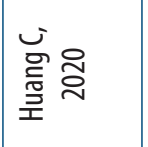 & 离 & 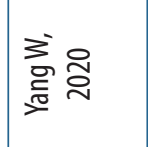 & 폴요 & 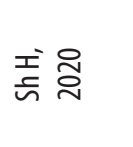 & 空 & خે̀ & 永 命 & Ð゙ すి & 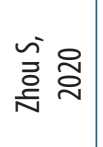 & 究。 \\
\hline \multicolumn{2}{|c|}{$\ddot{\Psi}$} & $\sigma$ & $\Phi$ & & 믄 & $\Xi$ & $\Xi$ & $\overline{\underline{e}}$ & $\Xi$ & $\underline{\varrho}$ & $\stackrel{\sigma}{\varrho}$ & $\Xi$ & $\Phi$ \\
\hline
\end{tabular}




\begin{tabular}{|c|c|c|c|c|c|c|c|c|c|c|c|}
\hline \multicolumn{2}{|l|}{$\begin{array}{l}\text { 音 } \\
\text { 言 }\end{array}$} & $r$ & $\infty$ & $r$ & $\infty$ & $\infty$ & $\infty$ & $\infty$ & $\sim$ & $\sim$ & $r$ \\
\hline \multirow{3}{*}{ 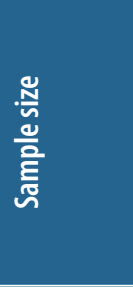 } & $\stackrel{I}{E}$ & 옹 & ㅁ & & $\approx$ & $\infty$ & & $\bar{\sim}$ & $\approx$ & $\stackrel{\circ}{\sim}$ & \\
\hline & 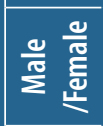 & 辜 & $\stackrel{ }{0}$ & 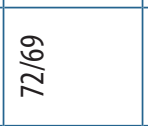 & $\stackrel{\operatorname{nn}}{\beth}$ & 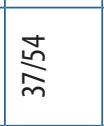 & $\stackrel{\circ}{\circ}$ & $\stackrel{ }{\stackrel{ }{I}}$ & $\stackrel{\unrhd}{\cong}$ & $\underset{\Im}{\stackrel{\infty}{\Im}}$ & $\frac{8}{6}$ \\
\hline & $\bar{\varepsilon}$ & gे & ㅇ & $\bar{\Xi}$ & $\approx$ & $\infty$ & $\stackrel{\infty}{\simeq}$ & $\bar{\sim}$ & $\stackrel{m}{\circ}$ & வ & $\hat{m}$ \\
\hline \multicolumn{2}{|l|}{ 흘 } & 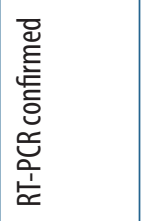 & 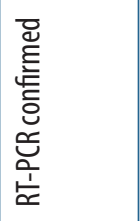 & 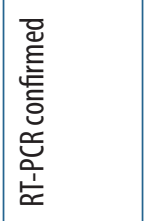 & 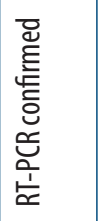 & 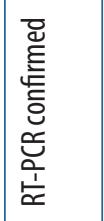 & 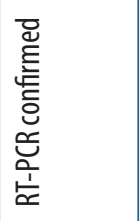 & 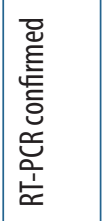 & 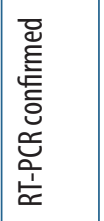 & 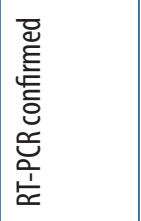 & 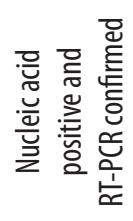 \\
\hline \multicolumn{2}{|l|}{ 产 } & 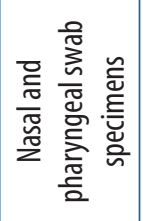 & 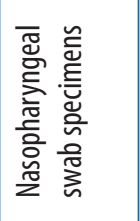 & 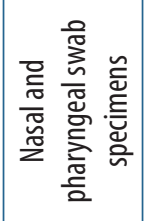 & 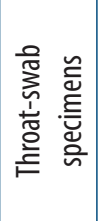 & 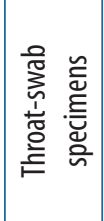 & 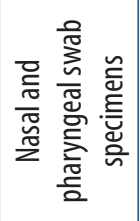 & 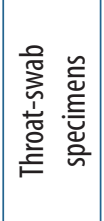 & 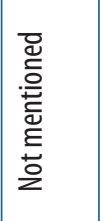 & 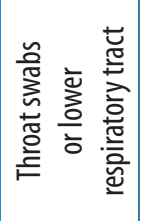 & 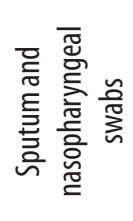 \\
\hline \multicolumn{2}{|l|}{ 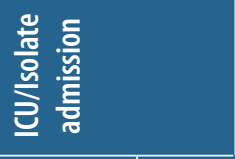 } & 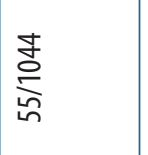 & & & & & & & & & \\
\hline \multirow{2}{*}{ 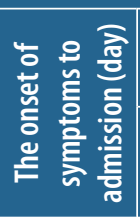 } & \begin{tabular}{|l|} 
产 \\
言
\end{tabular} & & & & $\overline{\bar{n}}$ & & & & & $\sigma$ & \\
\hline & 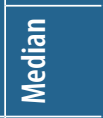 & & & & $\circ$ & & & & & $\stackrel{\circ}{\Gamma}$ & \\
\hline \multirow{2}{*}{ 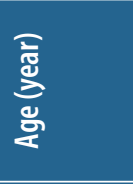 } & ทิ & & & & & & & & ț & 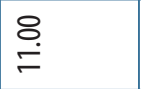 & 通 \\
\hline & 产 & & & $\stackrel{\circ}{+}$ & & & & & $\begin{array}{l}\text { तె } \\
\text { ல் }\end{array}$ & $\stackrel{\circ}{\dot{f}}$ & 웅 \\
\hline \multicolumn{2}{|l|}{ 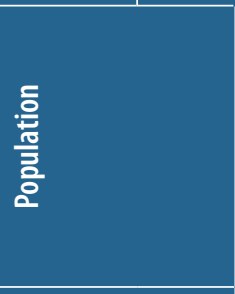 } & 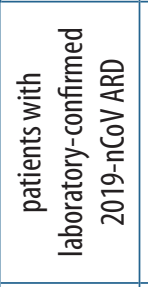 & 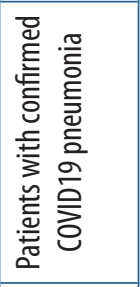 & 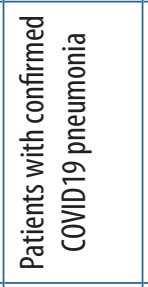 & 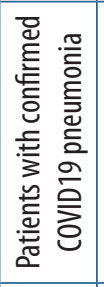 & 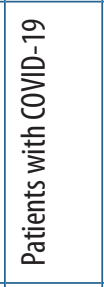 & 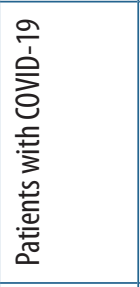 & 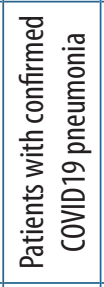 & 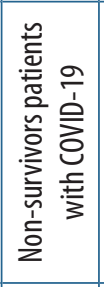 & 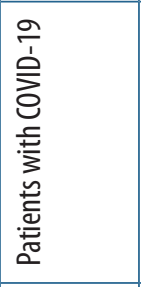 & 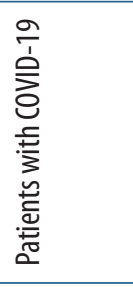 \\
\hline \multicolumn{2}{|l|}{ 产 } & 胥 & 突离总 & 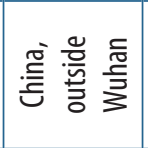 & 离胥 & 莣昜 & 哭离 & 突 & 离爱 & 哭 & 胥离 \\
\hline \multicolumn{2}{|l|}{ 咅 } & 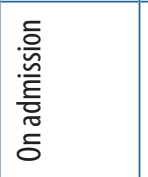 & 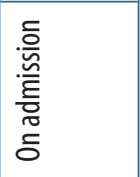 & 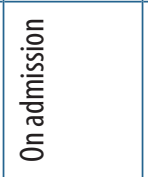 & 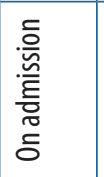 & 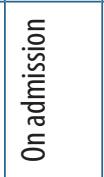 & 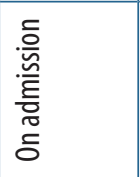 & 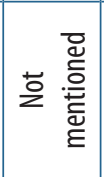 & 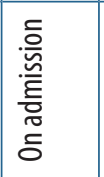 & 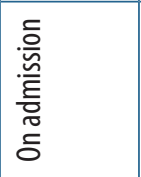 & 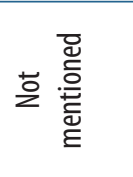 \\
\hline \multicolumn{2}{|l|}{ 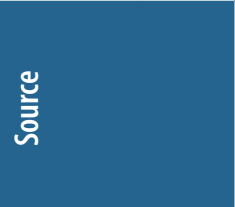 } & 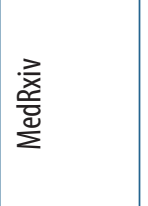 & 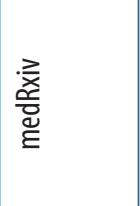 & 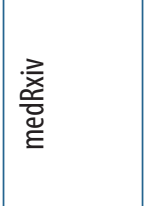 & 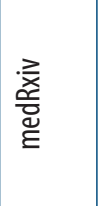 & 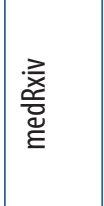 & 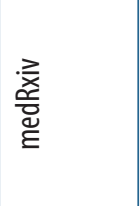 & 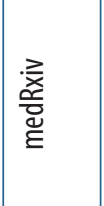 & 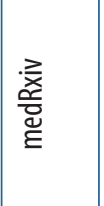 & 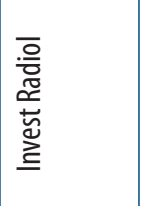 & 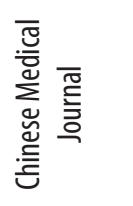 \\
\hline \multicolumn{2}{|c|}{ 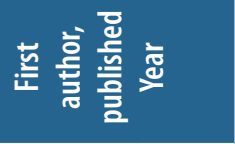 } & 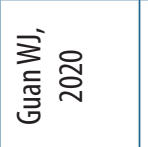 & 玄孞 & స్ㅎㅀㅀ & 产 京 & 灾 & 충 & 층요 & 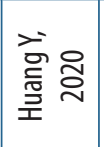 & 害古 & 字离 \\
\hline \multicolumn{2}{|c|}{ 酗 } & $\bar{\Xi}$ & 㐫 & $\bar{\Xi}$ & $\Xi$ & $\bar{\Xi}$ & $\Xi$ & $\Xi$ & $\underset{d}{\beth}$ & $\Xi$ & $\stackrel{\bar{d}}{d}$ \\
\hline
\end{tabular}




\begin{tabular}{|c|c|c|c|c|c|c|c|c|c|}
\hline \multicolumn{2}{|c|}{$\frac{3}{\frac{3}{2}}$} & $r$ & $\infty$ & $\infty$ & $a$ & $\infty$ & $\infty$ & $\infty$ & $a$ \\
\hline \multirow{3}{*}{ 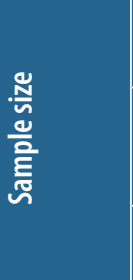 } & $\stackrel{I}{E}$ & $\stackrel{\infty}{=}$ & in & g & $=$ & & $\stackrel{\sharp}{m}$ & $\widetilde{\sigma}$ & $\neq$ \\
\hline & 产 & $\stackrel{\infty}{m}$ & 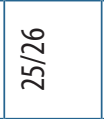 & $\frac{8}{6}$ & $\bar{\Xi}$ & 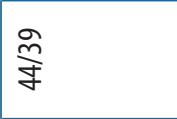 & $\frac{\widehat{0}}{\circ}$ & & $\stackrel{\infty}{\infty}$ \\
\hline & $\bar{\varepsilon}$ & $\bar{\sim}$ & $\bar{n}$ & $\bar{\beth}$ & $\bar{\sim}$ & $\approx$ & $\stackrel{\stackrel{m}{m}}{\text { s. }}$ & $\bar{n}$ & $\stackrel{\sim}{ }$ \\
\hline \multicolumn{2}{|l|}{ 을 } & 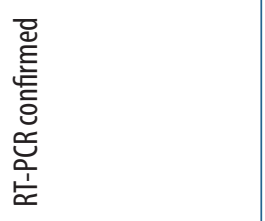 & 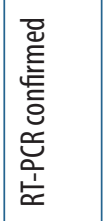 & 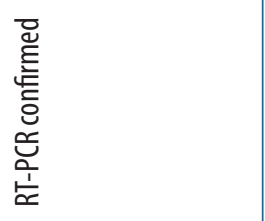 & 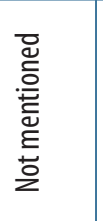 & 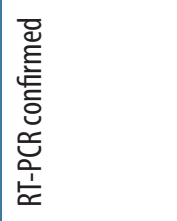 & 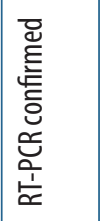 & 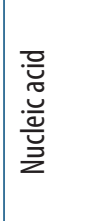 & 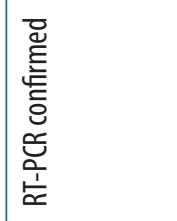 \\
\hline \multicolumn{2}{|l|}{ 哭 } & 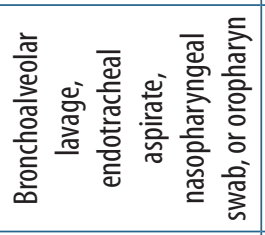 & 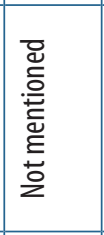 & 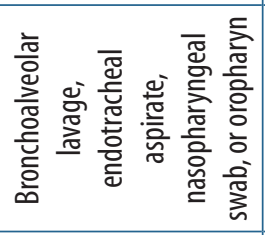 & 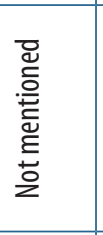 & 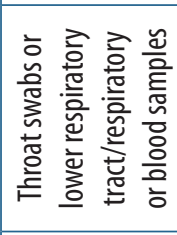 & 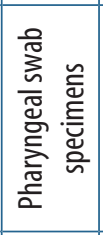 & 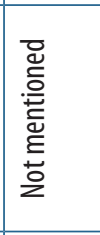 & 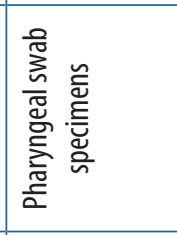 \\
\hline \multicolumn{2}{|l|}{ 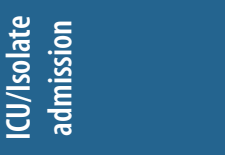 } & & & & & & & & $\stackrel{\approx}{=}$ \\
\hline \multirow{2}{*}{ 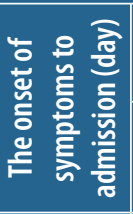 } & \begin{tabular}{|l|} 
๕ \\
๕
\end{tabular} & & $\frac{\vec{I}}{\check{I}}$ & $\stackrel{n}{I}$ & & & $\bar{\sigma}$ & & \\
\hline & 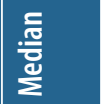 & & $\stackrel{\circ}{+}$ & & & & $\stackrel{\infty}{\circ}$ & & \\
\hline \multirow{2}{*}{ 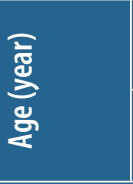 } & ทิ & $\begin{array}{l}\stackrel{8}{+} \\
\stackrel{+}{+}\end{array}$ & $\odot$ & هi & $\stackrel{\circ}{\check{x}}$ & $\stackrel{\mathscr{m}}{\check{I}}$ & & & \\
\hline & 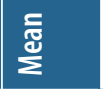 & $\stackrel{\circ}{\check{n}}$ & 염 & 욤 & $\bar{g}$ & $\stackrel{n}{q}$ & & & \\
\hline \multicolumn{2}{|l|}{ 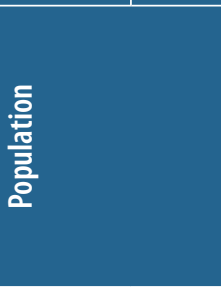 } & 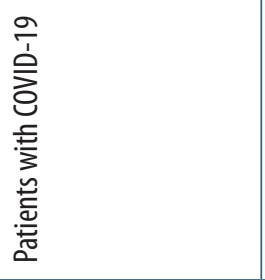 & 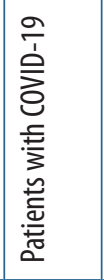 & 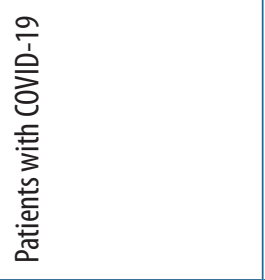 & 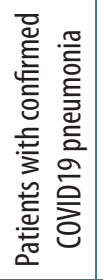 & 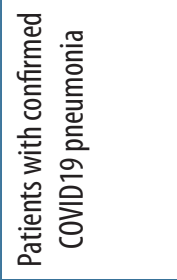 & 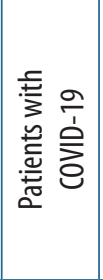 & 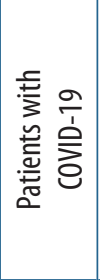 & 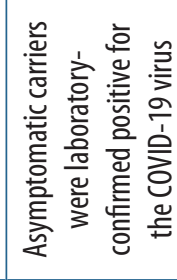 \\
\hline \multicolumn{2}{|l|}{ 흘 } & 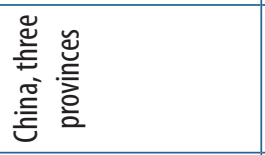 & 离总 & 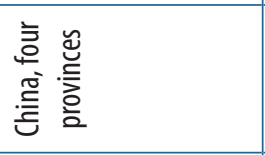 & 惫奇 & 总 & 兽总 & 离 总 & 离 \\
\hline \multicolumn{2}{|l|}{ 声苾 } & 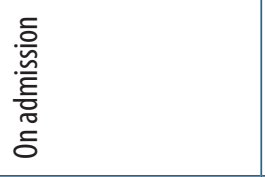 & 를 & 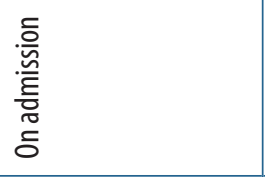 & 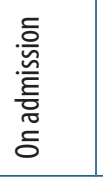 & 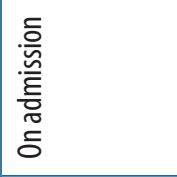 & 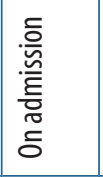 & 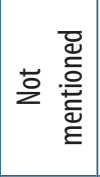 & 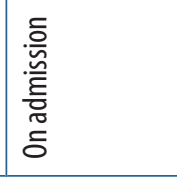 \\
\hline \multicolumn{2}{|l|}{ ڤ્气 } & $\begin{array}{l}\text { 흥 } \\
\text { 응 } \\
\text { वृ }\end{array}$ & $\begin{array}{l}\text { 흥 } \\
\text { 응 } \\
\text { 뚜 }\end{array}$ & $\begin{array}{l}\text { 흥 } \\
\text { 응 } \\
\text { 뚱 }\end{array}$ & 䓌 & 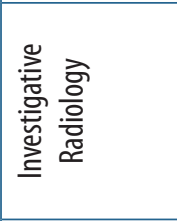 & 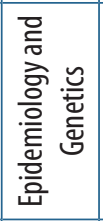 & 䔍 & 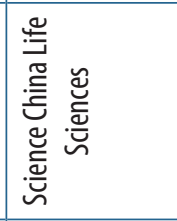 \\
\hline \multicolumn{2}{|c|}{ 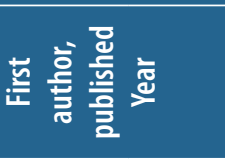 } & 离芯 & 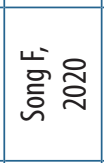 & 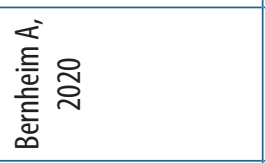 & 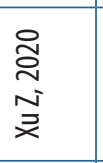 & 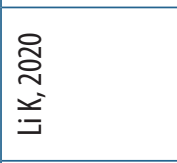 & 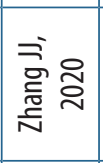 & 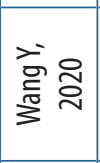 & $\begin{array}{l}\text { 岌 } \\
\text { N } \\
\text { 포 }\end{array}$ \\
\hline \multicolumn{2}{|c|}{ 芯 } & হ̄ & 户্户 & $\overline{\bar{m}}$ & $\widetilde{\widetilde{d}}$ & $\bar{m}$ & F & 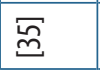 & $\bar{\varnothing}$ \\
\hline
\end{tabular}




\begin{tabular}{|c|c|c|c|c|c|c|c|c|c|c|c|}
\hline \multicolumn{2}{|l|}{ 蕞 } & $\infty$ & $r$ & $\infty$ & $r$ & $\infty$ & 0 & $r$ & $r$ & $\infty$ & $\infty$ \\
\hline \multirow{3}{*}{ 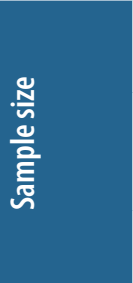 } & I & $\approx$ & $\stackrel{\circ}{\circ}$ & $\stackrel{\infty}{\curvearrowright}$ & $i n$ & $\stackrel{m}{m}$ & $\stackrel{\check{\sigma}}{\sigma}$ & 이 & 8 & $\stackrel{\infty}{\circ}$ & $F$ \\
\hline & 高 & $\stackrel{\mathbb{1}}{6}$ & $\frac{\infty}{\sigma}$ & 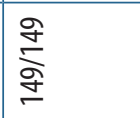 & 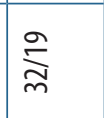 & $\stackrel{\varkappa}{\delta}$ & $\frac{\hat{a}}{\frac{a}{0}}$ & 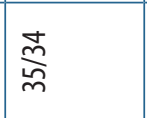 & $\frac{\hat{m}}{\mathrm{f}}$ & $\underset{\sim}{\stackrel{⿰}{\infty}}$ & 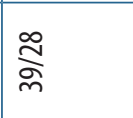 \\
\hline & $\bar{\varepsilon}$ & $\stackrel{\infty}{n}$ & $=$ & $\stackrel{\infty}{\infty}$ & in & $\stackrel{m}{m}$ & $\stackrel{\infty}{\circ}$ & o & $\bar{\circ}$ & t & 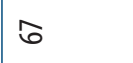 \\
\hline \multicolumn{2}{|l|}{ 을 } & 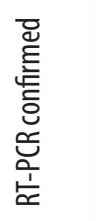 & 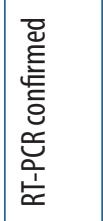 & 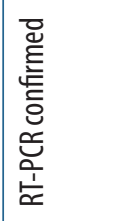 & 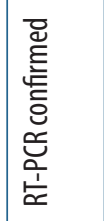 & 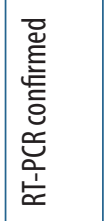 & 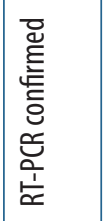 & 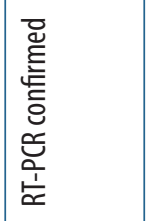 & 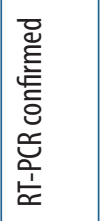 & 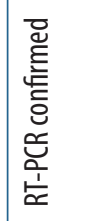 & 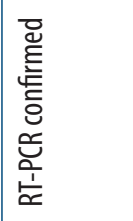 \\
\hline \multicolumn{2}{|l|}{ 哭 } & 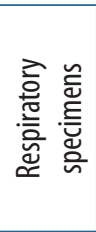 & 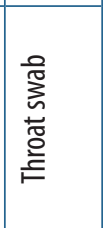 & 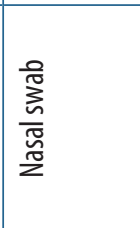 & 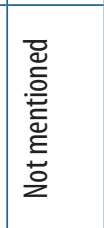 & 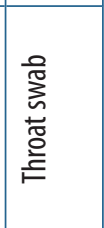 & 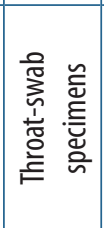 & 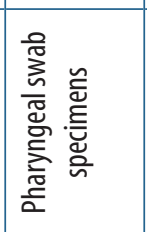 & 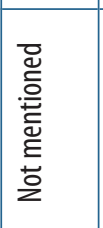 & 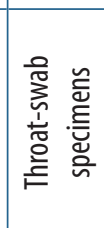 & 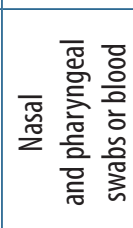 \\
\hline \multicolumn{2}{|l|}{ 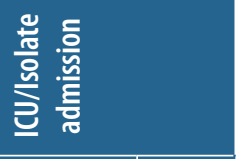 } & $\underset{\sim}{\stackrel{\sim}{\sim}}$ & $\underset{\substack{q \\
\infty}}{\stackrel{O}{\infty}}$ & 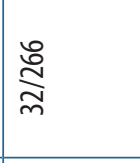 & $\frac{8}{N}$ & & $\frac{\mathfrak{g}}{\mathfrak{a}}$ & 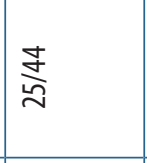 & 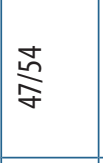 & & $\frac{8}{1}$ \\
\hline \multirow{2}{*}{ 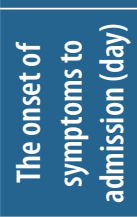 } & 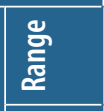 & & $\hat{i}$ & & $\stackrel{\infty}{m}$ & & & & \begin{tabular}{|l}
$n$ \\
$m$ \\
$m$ \\
$\infty$ \\
$\infty$
\end{tabular} & $\stackrel{\dot{m}}{\dot{\sigma}} \stackrel{0}{\dot{m}}$ & \\
\hline & 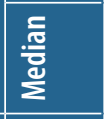 & & 울 & & 웅 & & 우 & & $\stackrel{\circ}{\check{E}}$ & $\stackrel{n}{\infty}$ & \\
\hline \multirow{2}{*}{ 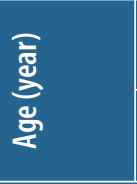 } & ถิ & & & $\begin{array}{l}\bar{\sigma} \\
\stackrel{m}{\nu}\end{array}$ & & $\stackrel{\sim}{\rightleftharpoons}$ & nీ & & $\frac{\pi}{a}$ & & $\stackrel{m}{\infty}$ \\
\hline & 宽 & & & 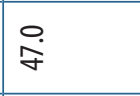 & & $\frac{n}{6}$ & in & & $\begin{array}{l}0 \\
\text { ơ } \\
\text { : }\end{array}$ & & $\stackrel{m}{m}$ \\
\hline \multicolumn{2}{|l|}{$\begin{array}{l}\text { 흘 } \\
\text { 을 } \\
\text { 흥 }\end{array}$} & 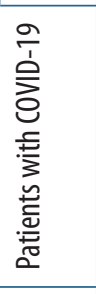 & 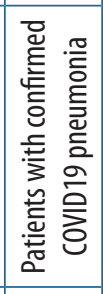 & 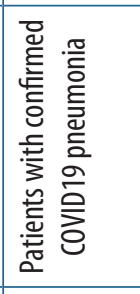 & 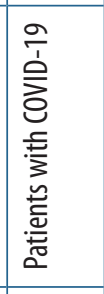 & 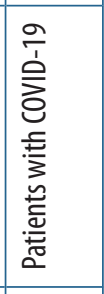 & 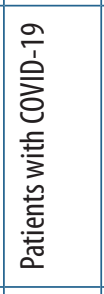 & 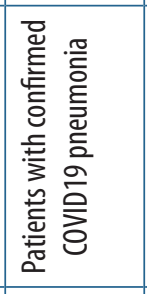 & 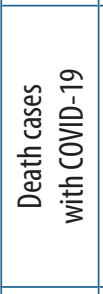 & 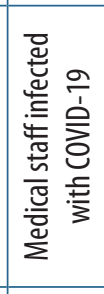 & 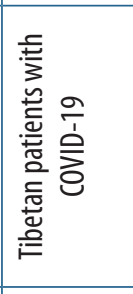 \\
\hline \multicolumn{2}{|l|}{ 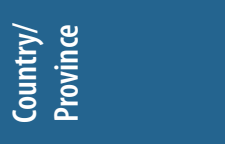 } & 预 & & 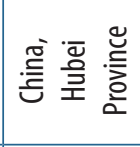 & 氦 & 蛋 总 & 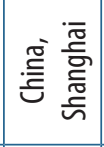 & 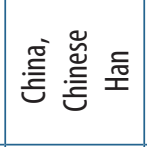 & 胥爱 & 疍胥 & 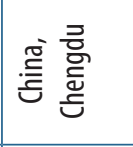 \\
\hline \multicolumn{2}{|l|}{ 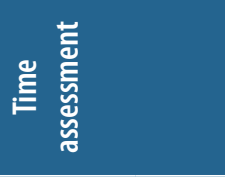 } & 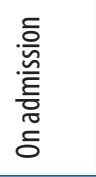 & 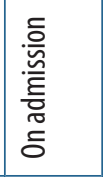 & 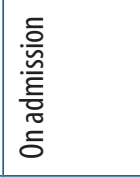 & 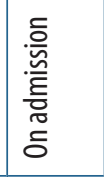 & 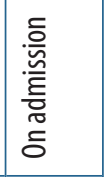 & 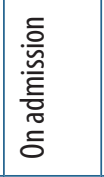 & 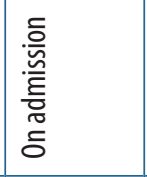 & 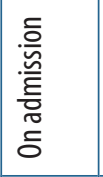 & 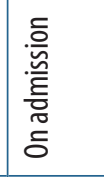 & 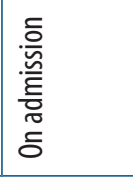 \\
\hline \multicolumn{2}{|l|}{ 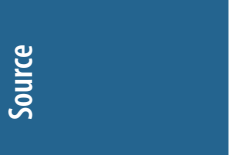 } & 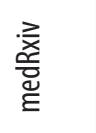 & 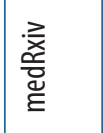 & 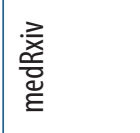 & 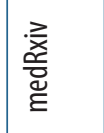 & 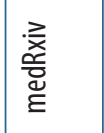 & 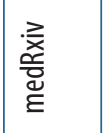 & 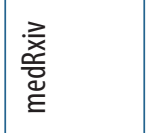 & 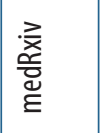 & 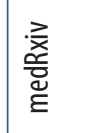 & 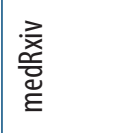 \\
\hline \multicolumn{2}{|c|}{ 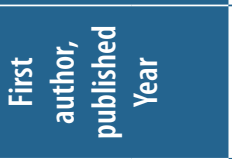 } & 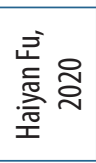 & 离 & 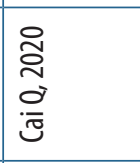 & 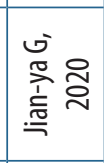 & 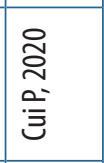 & $\begin{array}{l}\text { Dิ } \\
\text { Dิ } \\
\text { i } \\
\text { ్ }\end{array}$ & 离 & 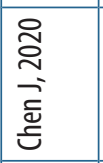 & 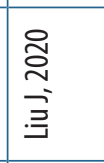 & 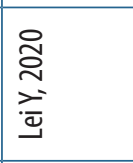 \\
\hline \multicolumn{2}{|l|}{ 芯 } & $\overline{\tilde{m}}$ & 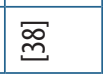 & ब్ & 定 & $\bar{\Xi}$ & $\bar{\Xi}$ & $\bar{\gamma}$ & F & 焉 & 脢 \\
\hline
\end{tabular}




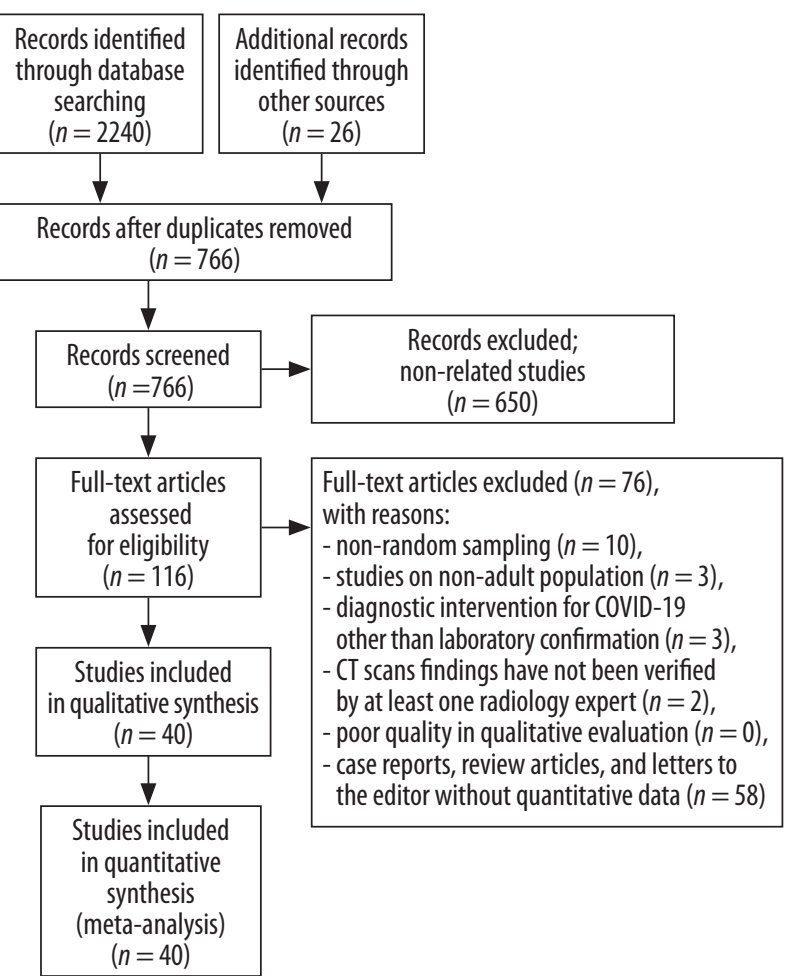

Figure 1. Preferred reporting items for systematic reviews and meta-analyses flowchart

35.7-62.8), 30.3\% (95\% CI: 19.6-43.6), 17.0\% (95\% CI: 3.9-50.9) and $16.6 \%$ (95\% CI: 13.6-20.2) of patients (Figure 3$)$.

\section{Other chest CT scan features}

Other chest CT scan features are shown in Figure 4: thickened interlobular septa - 63.6\% (95\% CI: 52.1-73.8), vascular enlargement - 61.4\% (95\% CI: 40.4-79.0), air bronchogram sign - 53.5\% (95\% CI: 40.3-66.2), bronchial wall thickening - 19.8\% (95\% CI: 12.6-29.6), bronchiolectasis - 19.9\% (95\% CI: 6.5-47.2), fibrous stripes - 17.2\% (95\% CI: 5.2-44.2), crazy-paving pattern - 21.7\% (95\% CI: $13.8-$ $32.5)$, thickening of the adjacent pleura $-30.0 \%$ (95\% CI: 16.1-48.8), pleural effusion - 6.9\% (95\% CI: 4.7-10.1) and lymphadenopathy - 4.7\% (95\% CI: 3.0-7.5) (Figure 4).

\section{Lesions distribution}

The distribution of lung lesions in patients with COVID-19 pneumonia was as follows: peripheral (70.0\% [95\% CI: 57.8-79.9]), central (3.9\% [95\% CI: 1.4-10.6]), and peripheral and central (31.1\% [95\% CI: 19.5-45.8]) (Suppl Figure 1).

\section{Lobes involvement}

Pulmonary lobes involvement in patients with COVID-19 pneumonia was as follows (Figure 5): right upper lobe
(58.4\% [95\% CI: 33.6-79.5]), right middle lobe (49.7\% [95\% CI: 23.0-76.6]), right lower lobe (86.5\% [95\% CI: 57.7-96.8]), left upper lobe (64.5\% [95\% CI: 37.3-84.7]), and left lower lobe (81.0\% [95\% CI: 50.5-94.7]).

\section{Number of involved lobes}

In patients with COVID-19 pneumonia, the number of involved lobes was as follows (Suppl Figure 2): one lobe (58.4\% [95\% CI: 33.6-79.5]), two lobes (31.1\% [95\% CI: 19.5-45.8]), three lobes (10.5\% [95\% CI: 8.0-13.8]), four lobes (20.1\% [95\% CI: 15.4-25.9]), and five lobes (43.2\% [95\% CI: 34.2-52.6]).

\section{Sensitivity analysis}

Sensitivity analysis was performed for all meta-analyses and it showed that the overall result remains robust after the omission of one study at a time (Suppl Figures 3-7).

\section{Risk of publication bias}

Publication bias was evaluated for studies that showed positive chest CT scan of COVID-19 patients (Begg's test $=0.774$ and Egger's test $<0.001$ ) and for studies that showed bilateral lung involvement in chest CT scan of COVID-19 patients pneumonia (Begg's test $=0.194$ and Egger's test < 0.001) (Suppl Figure 8).

\section{Discussion}

The present study is the first systematic review and metaanalysis that extensively examines chest CT scan findings in COVID-19 patients. Our study showed that chest CT scan has high sensitivity in the diagnosis of COVID-19. COVID-19 is a new disease with serious consequences for public health. Chest CT scan is an important part of disease detection for patients suspected of having COVID-19 infection and may help early detection of lung malformations for the purpose of screening highly suspected patients, especially those with negative initial reverse-transcription polymerase chain reaction (RT-PCR) screening [54]. In fact, given the limited number of RT-PCR kits in many centers and the likelihood of false negative RT-PCR results, the National Health Commission of China has encouraged clinical findings and chest CT scan [55]. Our review showed some imaging findings that are often seen in patients with COVID-19. The present study showed that 94.5\% of COVID-19 patients had positive chest CT scan findings, while the frequency of bilateral lung involvement in chest CT scan of COVID-19 pneumonia patients was $79.1 \%$. It is important to observe the high incidence of bilateral organizing pneumonia in these patients. This suggests that corticosteroids may be an option to suppress this immune response in lung parenchyma of COVID-19 pneumonia. 
A Study name

Chen G, 2020

Huang C, 2020

Chen N, 2020

Yang W, 2020

Xu Y H, 2020

$\mathrm{Sh} \mathrm{H}, 2020$

Wang D, 2020

Pan Y, 2020

XuX, 2020

Li Y, 2020

Zhou S, 2020

Zhao W, 2020

Guan W. J, 2020

Ai J. W, 2020

Yuan M, 2020

Qian G. Q, 2020

Liang Y, 2020

Huang $Y, 2020$

Wu J, 2020

Chung M, 2020

Song F, 2020

Bernheim A, 2020

Xu Z, 2020

Zhang JJ, 2020

Wang Y, 2020

Hu Z, 2020

Haiyan Fu, 2020

Li」, 2020

Cai Q, 2020

Jian-ya G, 2020

Cui P, 2020

Cao M, 2020

Xu Y, 2020

Chen J, 2020

Liu J, 2020

Lei Y, 2020
Statistics for each study

\begin{tabular}{|c|c|c|c|c|}
\hline $\begin{array}{c}\text { Event } \\
\text { rate }\end{array}$ & $\begin{array}{c}\text { Lower } \\
\text { limit }\end{array}$ & $\begin{array}{c}\text { Upper } \\
\text { limit }\end{array}$ & Z-Value & p-Value \\
\hline 0.977 & 0.723 & 0.999 & 2.629 & 0.009 \\
\hline 0.988 & 0.836 & 0.999 & 3.106 & 0.002 \\
\hline 0.995 & 0.925 & 1.000 & 3.734 & 0.000 \\
\hline 0.886 & 0.824 & 0.928 & 7.954 & 0.000 \\
\hline 0.820 & 0.689 & 0.904 & 4.119 & 0.000 \\
\hline 0.994 & 0.910 & 1.000 & 3.591 & 0.000 \\
\hline 0.996 & 0.945 & 1.000 & 3.970 & 0.000 \\
\hline 0.992 & 0.887 & 1.000 & 3.412 & 0.001 \\
\hline 0.984 & 0.894 & 0.998 & 4.078 & 0.000 \\
\hline 0.990 & 0.864 & 0.999 & 3.261 & 0.001 \\
\hline 0.992 & 0.885 & 1.000 & 3.401 & 0.001 \\
\hline 0.921 & 0.850 & 0.960 & 6.658 & 0.000 \\
\hline 0.764 & 0.738 & 0.788 & 16.554 & 0.000 \\
\hline 0.976 & 0.713 & 0.999 & 2.594 & 0.009 \\
\hline 0.982 & 0.770 & 0.999 & 2.808 & 0.005 \\
\hline 0.943 & 0.871 & 0.976 & 6.101 & 0.000 \\
\hline 0.977 & 0.723 & 0.999 & 2.629 & 0.009 \\
\hline 0.889 & 0.739 & 0.958 & 3.921 & 0.000 \\
\hline 0.950 & 0.874 & 0.981 & 5.740 & 0.000 \\
\hline 0.857 & 0.639 & 0.953 & 2.873 & 0.004 \\
\hline 0.980 & 0.874 & 0.997 & 3.873 & 0.000 \\
\hline 0.777 & 0.694 & 0.842 & 5.713 & 0.000 \\
\hline 0.810 & 0.588 & 0.927 & 2.604 & 0.009 \\
\hline 0.993 & 0.949 & 0.999 & 4.880 & 0.000 \\
\hline 0.963 & 0.942 & 0.976 & 13.918 & 0.000 \\
\hline 0.708 & 0.502 & 0.854 & 1.976 & 0.048 \\
\hline 0.611 & 0.446 & 0.754 & 1.322 & 0.186 \\
\hline 0.941 & 0.680 & 0.992 & 2.690 & 0.007 \\
\hline 0.998 & 0.974 & 1.000 & 4.516 & 0.000 \\
\hline 0.990 & 0.864 & 0.999 & 3.261 & 0.001 \\
\hline 0.986 & 0.813 & 0.999 & 2.993 & 0.003 \\
\hline 0.985 & 0.954 & 0.995 & 7.175 & 0.000 \\
\hline 0.993 & 0.896 & 1.000 & 3.477 & 0.001 \\
\hline 0.980 & 0.924 & 0.995 & 5.463 & 0.000 \\
\hline 0.906 & 0.807 & 0.957 & 5.290 & 0.000 \\
\hline 0.701 & 0.582 & 0.799 & 3.200 & 0.001 \\
\hline 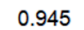 & 0.917 & 0.963 & 12.784 & 0.000 \\
\hline
\end{tabular}

Event rate and $95 \% \mathrm{Cl}$

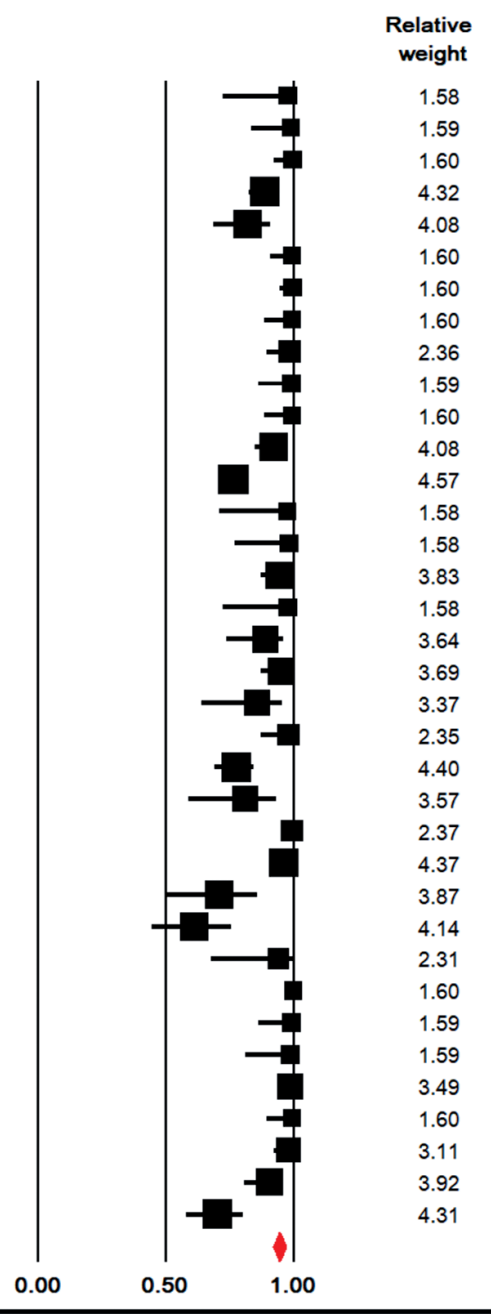

Heterogeneity: $\mathrm{I}^{2}=\mathbf{9 8 . 6 \%}, \mathrm{P}<0.001$

Meta Analysis

\begin{tabular}{|c|c|c|c|c|c|c|c|}
\hline \multirow[t]{2}{*}{ Study name } & \multirow[b]{2}{*}{$\begin{array}{c}\text { Event } \\
\text { rate }\end{array}$} & \multicolumn{3}{|c|}{ Statistics for each study } & \multirow[b]{2}{*}{$\mathrm{p}$-Value } & \multirow[t]{2}{*}{ Event rate and $95 \% \mathrm{Cl}$} & \multirow[b]{2}{*}{$\begin{array}{l}\text { Relative } \\
\text { weight }\end{array}$} \\
\hline & & $\begin{array}{c}\text { Lower } \\
\text { limit }\end{array}$ & $\begin{array}{l}\text { Upper } \\
\text { limit }\end{array}$ & Z-Value & & & \\
\hline Chen G, 2020 & 0.810 & 0.588 & 0.927 & 2.604 & 0.009 & & 4.14 \\
\hline Huang C, 2020 & 0.976 & 0.846 & 0.997 & 3.644 & 0.000 & & 2.28 \\
\hline Chen N, 2020 & 0.758 & 0.664 & 0.832 & 4.859 & 0.000 & & 5.83 \\
\hline Sh H, 2020 & 0.790 & 0.688 & 0.865 & 4.859 & 0.000 & & 5.65 \\
\hline Wang D, 2020 & 0.996 & 0.945 & 1.000 & 3.970 & 0.000 & & 1.41 \\
\hline Zhao W, 2020 & 0.892 & 0.812 & 0.941 & 6.322 & 0.000 & & 5.34 \\
\hline Guan W. J, 2020 & 0.601 & 0.568 & 0.634 & 5.825 & 0.000 & & 6.34 \\
\hline Ai J. W, 2020 & 0.600 & 0.380 & 0.786 & 0.888 & 0.374 & & 4.68 \\
\hline Feng Z, 2020 & 0.872 & 0.806 & 0.918 & 7.615 & 0.000 & & 5.75 \\
\hline Yuan M, 2020 & 0.852 & 0.665 & 0.943 & 3.229 & 0.001 & & 4.21 \\
\hline Qian G. Q, 2020 & 0.735 & 0.630 & 0.819 & 4.101 & 0.000 & & 5.77 \\
\hline Huang Y, 2020 & 0.969 & 0.809 & 0.996 & 3.380 & 0.001 & & 2.27 \\
\hline Kui L, 2020 & 0.847 & 0.776 & 0.898 & 7.207 & 0.000 & & 5.82 \\
\hline Chung M, 2020 & 0.889 & 0.648 & 0.972 & 2.773 & 0.006 & & 3.21 \\
\hline Song F, 2020 & 0.880 & 0.758 & 0.945 & 4.578 & 0.000 & & 4.79 \\
\hline Bernheim A, 2020 & 0.777 & 0.681 & 0.850 & 5.032 & 0.000 & & 5.77 \\
\hline Li K, 2020 & 0.952 & 0.879 & 0.982 & 5.821 & 0.000 & & 4.37 \\
\hline Zhang JJ, 2020 & 0.903 & 0.840 & 0.943 & 7.643 & 0.000 & & 5.56 \\
\hline Xu Y, 2020 & 0.623 & 0.504 & 0.729 & 2.025 & 0.043 & & 5.77 \\
\hline Liu J, 2020 & 0.672 & 0.543 & 0.780 & 2.570 & 0.010 & & 5.62 \\
\hline \multirow[t]{2}{*}{ Lei Y, 2020} & 0.702 & 0.558 & 0.815 & 2.688 & 0.007 & & 5.42 \\
\hline & 0.825 & 0.764 & 0.872 & 8.127 & 0.000 & & \\
\hline & & & & & & 0.50 & \\
\hline
\end{tabular}

\section{Meta Analysis}

Figure 2. Meta-analysis of sensitivity of chest computed tomography scan (A) and meta-analysis of bilateral lung involvement in chest computed tomography scan (B) in patients with COVID-19 
Study name

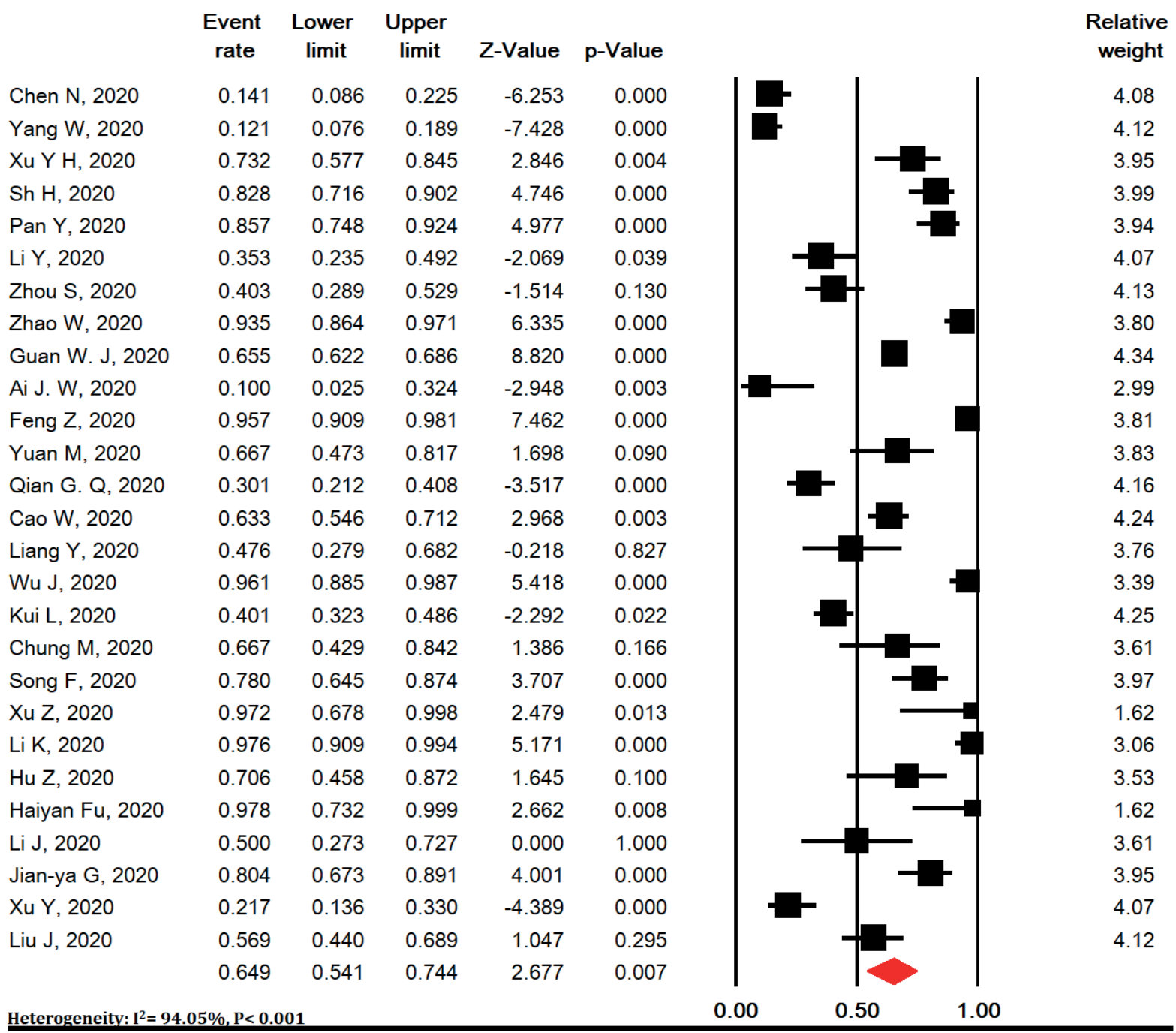

\section{Event rate and $95 \% \mathrm{Cl}$}

lative

4.12

3.99

.94

3.80

2.99

.81

4.16

2.24

3.39

3.97

1.62

3.53

1.62

3.95

4.07

12

\section{Meta Analysis}

B

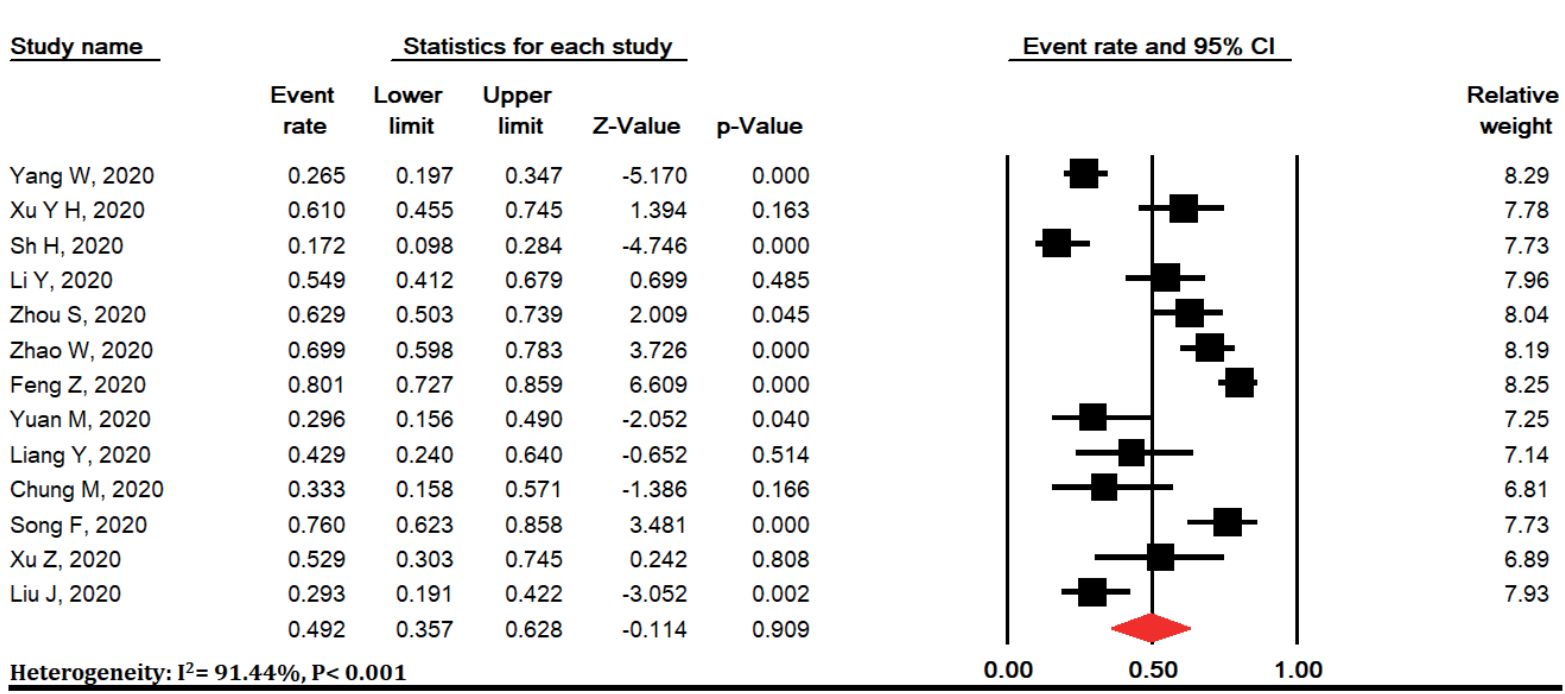
Meta Analysis

Figure 3. Meta-analysis of pure ground-glass opacity (GGO) (A), mixed (GGO pulse consolidation or reticular) (B), reticular (C), consolidation (D), and presence of nodule (E) findings in chest computed tomography scan of COVID-19 pneumonia 


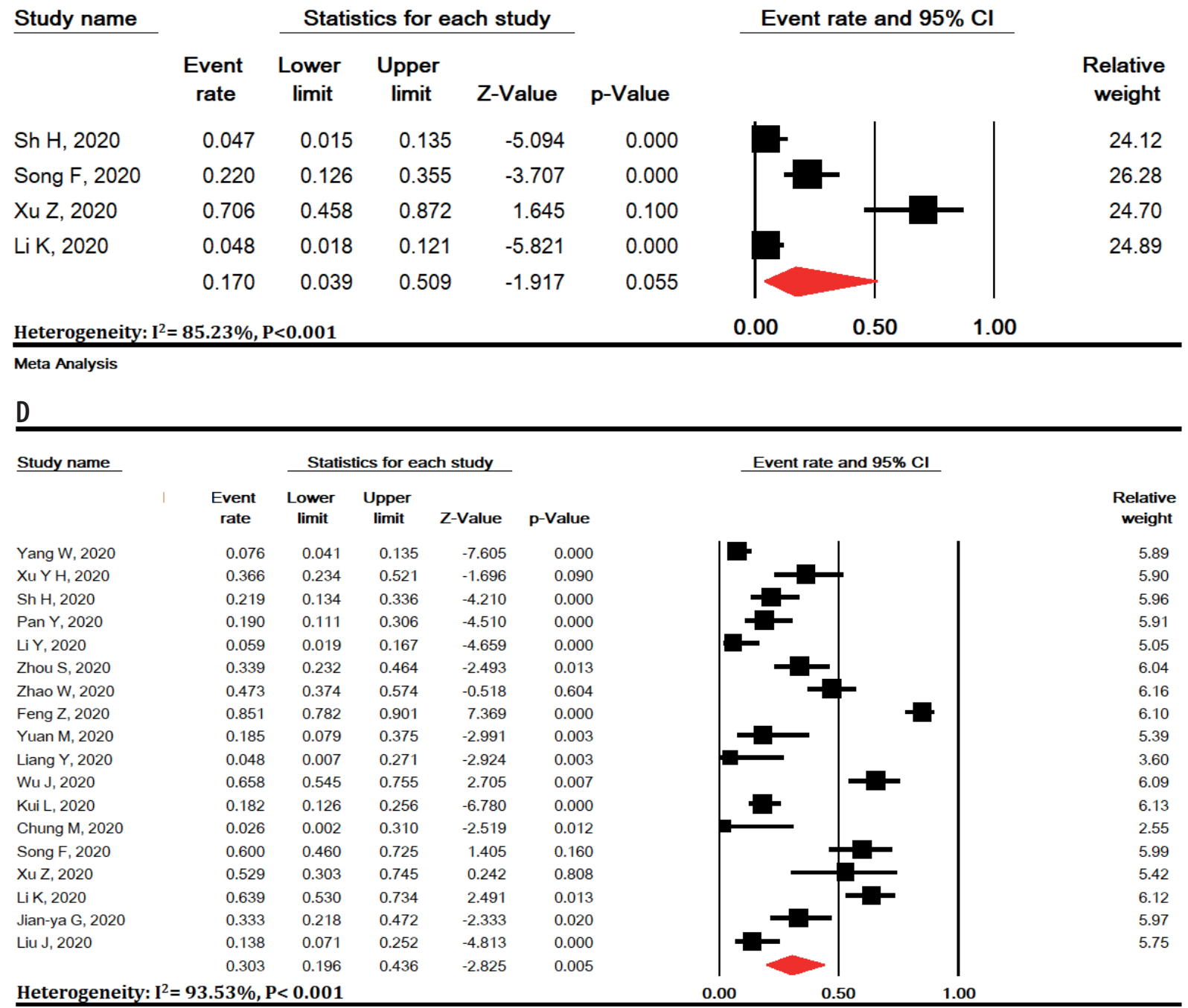

Meta Analysis

E

Study name

\begin{tabular}{|c|c|c|c|c|c|c|c|}
\hline & $\begin{array}{l}\text { Event } \\
\text { rate }\end{array}$ & $\begin{array}{l}\text { Lower } \\
\text { limit }\end{array}$ & $\begin{array}{l}\text { Upper } \\
\text { limit }\end{array}$ & Z-Value & p-Value & & $\begin{array}{r}\text { Relative } \\
\text { weight }\end{array}$ \\
\hline Sh H, 2020 & 0.078 & 0.033 & 0.174 & -5.299 & 0.000 & $=$ & 6.87 \\
\hline Pan Y, 2020 & 0.349 & 0.242 & 0.474 & -2.356 & 0.018 & & 21.34 \\
\hline Li Y, 2020 & 0.216 & 0.124 & 0.349 & -3.792 & 0.000 & & 12.86 \\
\hline Zhao W, 2020 & 0.247 & 0.170 & 0.345 & -4.631 & 0.000 & & 25.80 \\
\hline Feng Z, 2020 & 0.078 & 0.044 & 0.135 & -7.865 & 0.000 & & 15.11 \\
\hline Yuan M, 2020 & 0.074 & 0.019 & 0.252 & -3.437 & 0.001 & & 2.76 \\
\hline Liang Y, 2020 & 0.023 & 0.001 & 0.277 & -2.629 & 0.009 & & 0.73 \\
\hline Bernheim A, 2020 & 0.005 & 0.000 & 0.079 & -3.697 & 0.000 & E & 0.74 \\
\hline Li K, 2020 & 0.072 & 0.033 & 0.152 & -6.021 & 0.000 & - & 8.30 \\
\hline \multirow[t]{2}{*}{ Jian-ya G, 2020} & 0.078 & 0.030 & 0.191 & -4.731 & 0.000 & 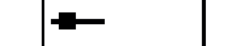 & 5.49 \\
\hline & 0.166 & 0.136 & 0.202 & -13.203 & 0.000 & & \\
\hline \multicolumn{6}{|c|}{ Heterogeneity: $\mathrm{I}^{2}=\mathbf{8 1 . 2 5} \%, \mathrm{P}<0.001$} & 0.00 & \\
\hline
\end{tabular}

Meta Analysis

Figure 3. Cont. 
A

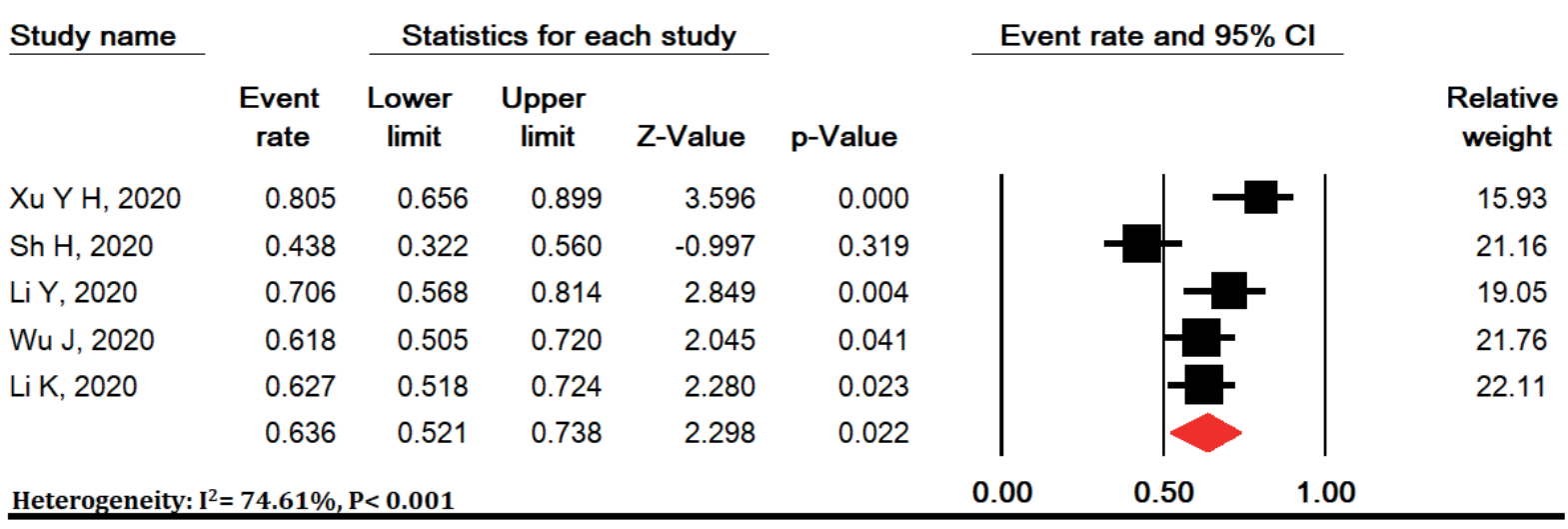

Meta Analysis

B

Study name

Statistics for each study

Event rate and $95 \% \mathrm{Cl}$

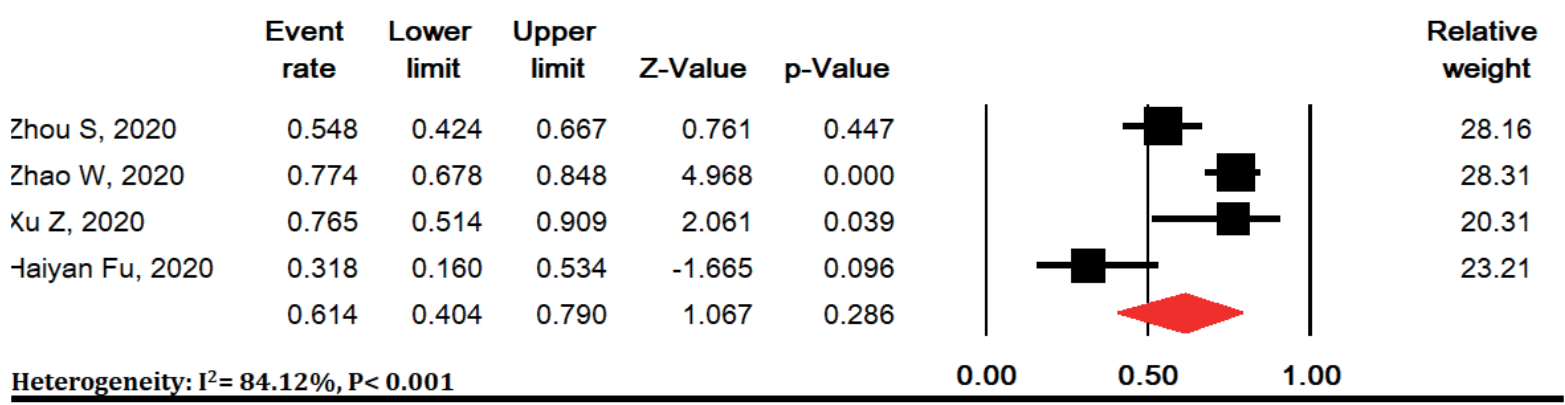

Meta Analysis

C

Study name

Statistics for each study

Event rate and $95 \% \mathrm{Cl}$

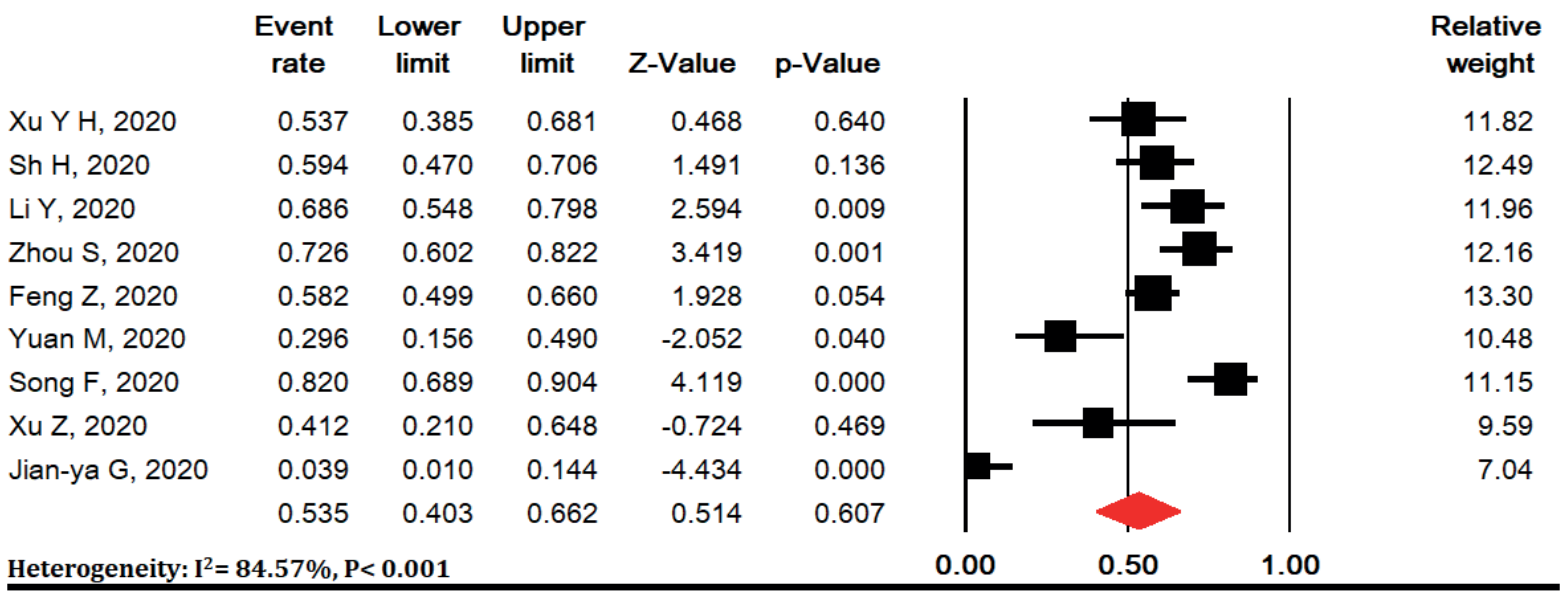

Meta Analysis

Figure 4. Meta-analysis of thickened interlobular septa (A), vascular enlargement (B), air bronchogram sign (C), bronchial wall thickening (D), bronchiolectasis $(\mathrm{E})$, fibrous stripes $(\mathrm{F})$, crazy-paving pattern $(\mathrm{G})$, thickening of the adjacent pleura $(\mathrm{H})$, pleural effusion $(\mathrm{I})$ and lymphadenopathy $(\mathrm{J})$ findings in chest computed tomography scan of COVID-19 pneumonia 
Study name

Zhao W, 2020
Wu J, 2020
Bernheim A, 2020
Li K, 2020

Statistics for each study

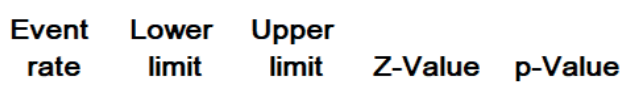

$\begin{array}{lllll}0.312 & 0.226 & 0.413 & -3.536 & 0.000 \\ 0.118 & 0.063 & 0.212 & -5.655 & 0.000 \\ 0.149 & 0.090 & 0.236 & -6.016 & 0.000 \\ 0.229 & 0.151 & 0.331 & -4.648 & 0.000 \\ 0.198 & 0.126 & 0.296 & -5.134 & 0.000\end{array}$

Event rate and $95 \% \mathrm{Cl}$
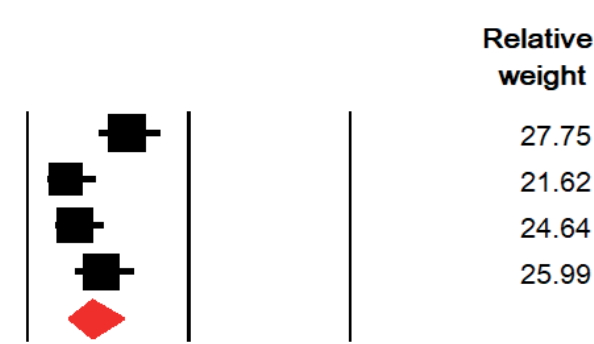

27.75

21.62

24.64

25.99

Heterogeneity: $\mathrm{I}^{2}=\mathbf{7 4 . 0 7} \%, \mathrm{P}=\mathbf{0 . 0 0 9}$

0.00 0.50 Meta Analysis E

Study name

$\begin{array}{lcccrr} & \begin{array}{c}\text { Event } \\ \text { rate }\end{array} & \begin{array}{c}\text { Lower } \\ \text { limit }\end{array} & \begin{array}{c}\text { Upper } \\ \text { limit }\end{array} & \text { Z-Value } & \text { p-Value } \\ \text { Sh H, 2020 } & 0.141 & 0.075 & 0.249 & -5.034 & 0.000 \\ \text { Zhou S, 2020 } & 0.323 & 0.219 & 0.448 & -2.731 & 0.006 \\ \text { Zhao W, 2020 } & 0.570 & 0.468 & 0.666 & 1.344 & 0.179 \\ \text { Bernheim A, 2020 } & 0.011 & 0.001 & 0.072 & -4.508 & 0.000 \\ & 0.199 & 0.065 & 0.472 & -2.132 & 0.033\end{array}$

Event rate and $95 \% \mathrm{Cl}$

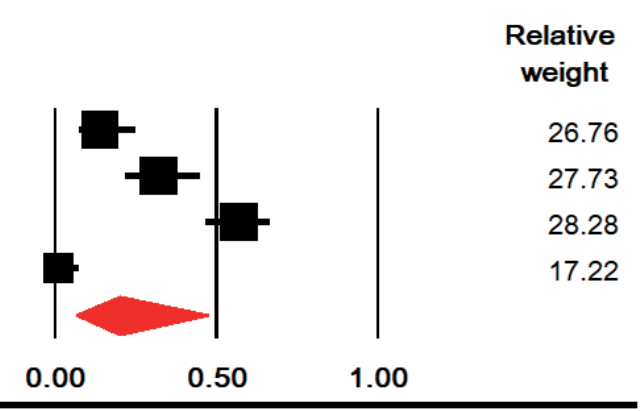

Heterogeneity: $\mathrm{I}^{2}=\mathbf{9 3 . 1 9} \%, \mathrm{P}<0.001$ Meta Analysis

F

Study name

$\begin{array}{lcccrr} & \begin{array}{c}\text { Event } \\ \text { rate }\end{array} & \begin{array}{c}\text { Lower } \\ \text { limit }\end{array} & \begin{array}{c}\text { Upper } \\ \text { limit }\end{array} & \text { Z-Value } & \text { p-Value } \\ \text { Pan Y, 2020 } & 0.175 & 0.099 & 0.288 & -4.681 & 0.000 \\ \text { Zhou S, 2020 } & 0.565 & 0.440 & 0.682 & 1.013 & 0.311 \\ \text { Chung M, 2020 } & 0.026 & 0.002 & 0.310 & -2.519 & 0.012 \\ \text { Bernheim A, 2020 } & 0.005 & 0.000 & 0.079 & -3.697 & 0.000 \\ \text { Xu Z, 2020 } & 0.353 & 0.168 & 0.596 & -1.194 & 0.232 \\ & 0.172 & 0.052 & 0.442 & -2.304 & 0.021\end{array}$

Event rate and $95 \% \mathrm{Cl}$

Relative weight

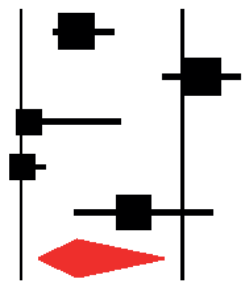

25.48

26.12

12.34

12.48

23.58

Heterogeneity: $\mathrm{I}^{2}=\mathbf{8 8 . 4 4 \%}, \mathrm{P}<\mathbf{0 . 0 0 1}$

0.00

0.50 1.00

Meta Analysis

G

Study name

$\begin{array}{lcccrr} & \begin{array}{c}\text { Event } \\ \text { rate }\end{array} & \begin{array}{c}\text { Lower } \\ \text { limit }\end{array} & \begin{array}{c}\text { Upper } \\ \text { limit }\end{array} & \text { Z-Value } & \text { p-Value } \\ \text { Sh H, 2020 } & 0.125 & 0.064 & 0.231 & -5.148 & 0.000 \\ \text { Feng Z, 2020 } & 0.298 & 0.228 & 0.378 & -4.656 & 0.000 \\ \text { Wu J, 2020 } & 0.303 & 0.210 & 0.414 & -3.343 & 0.001 \\ \text { Chung M, 2020 } & 0.222 & 0.086 & 0.465 & -2.210 & 0.027 \\ \text { Bernheim A, 2020 } & 0.064 & 0.029 & 0.135 & -6.365 & 0.000 \\ \text { Li K, 2020 } & 0.361 & 0.266 & 0.470 & -2.491 & 0.013 \\ & 0.217 & 0.138 & 0.325 & -4.556 & 0.000\end{array}$

Event rate and $95 \% \mathrm{Cl}$

Relative weight

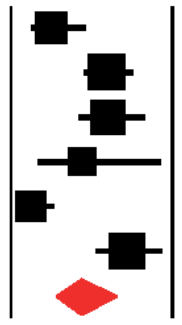

15.73

20.06

18.71

11.62

14.71

19.17

Heterogeneity: $\mathrm{I}^{2}=\mathbf{8 1 . 4 6 \%}, \mathrm{P}<0.001$

0.00

0.50 1.00

Meta Analysis

Figure 4. Cont. 
H

Study name

Statistics for each study

Event rate and $95 \% \mathrm{Cl}$

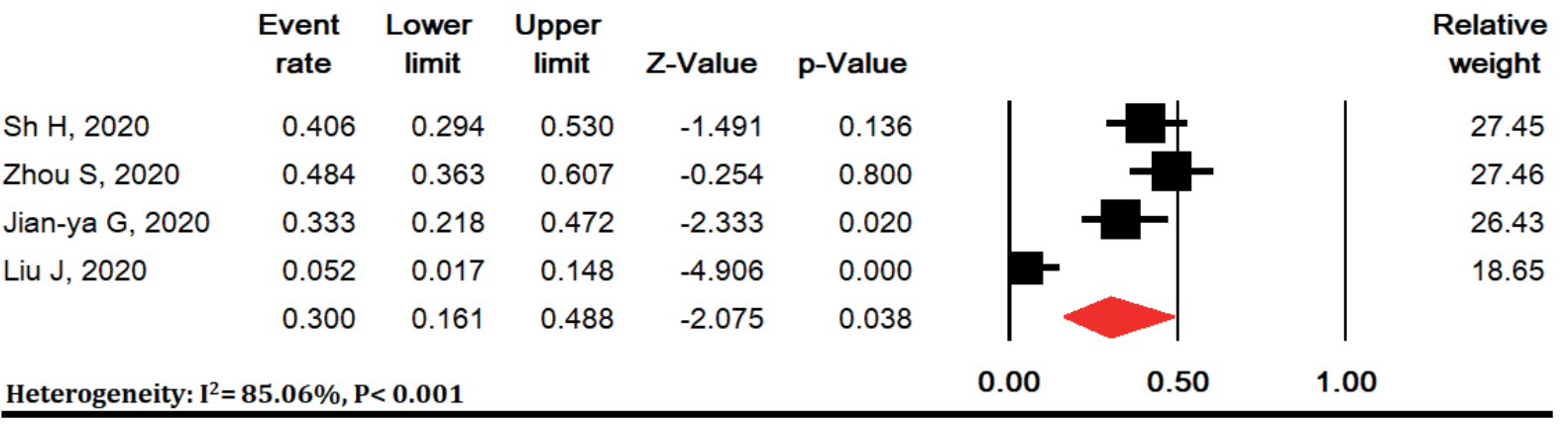

Meta Analysis

I

Study name

Xu Y H, 2020
Sh H, 2020
Li Y, 2020
Zhou S, 2020
Zhao W, 2020
Feng Z, 2020
Yuan M, 2020
Wu J, 2020
Song F, 2020
Bernheim A, 2020
Li K, 2020
Jian-ya G, 2020
Liu J, 2020

Statistics for each study

Event Lower Upper

rate limit limit

$0.098 \quad 0.037$

0.049

0.020

0.097

0.151

0.028

0.037

0.066

0.080

0.011

0.084

0.137

0.008

0.069
$0.037 \quad 0.233$

$0.019 \quad 0.124$

$0.003 \quad 0.126$

$0.044 \quad 0.199$

$0.091 \quad 0.238$

$0.011 \quad 0.073$

$0.005 \quad 0.221$

$0.028 \quad 0.149$

$0.030 \quad 0.195$

$0.001 \quad 0.072$

0.041

0.067

0.001

0.047
0.166

0.261

0.121

0.101
Z-Value

$$
-4.227
$$$$
-5.767
$$

$-3.873$

$-5.200$

$-5.967$

$-6.966$

$-3.197$

$-5.734$

$-4.685$

$-4.508$

$-6.038$

$-4.518$

$-3.353$

$-12.272$

Heterogeneity: $\mathrm{I}^{2}=\mathbf{5 0 . 4 0} \%, \mathrm{P}=0.019$

Meta Analysis

J

Study name

$\begin{array}{lcccrr} & \begin{array}{c}\text { Event } \\ \text { rate }\end{array} & \begin{array}{c}\text { Lower } \\ \text { limit }\end{array} & \begin{array}{c}\text { Upper } \\ \text { limit }\end{array} & \text { Z-Value } & \text { p-Value } \\ \text { Xu Y H, 2020 } & 0.024 & 0.003 & 0.154 & -3.644 & 0.000 \\ \text { Sh H, 2020 } & 0.078 & 0.033 & 0.174 & -5.299 & 0.000 \\ \text { Li Y, 2020 } & 0.010 & 0.001 & 0.136 & -3.261 & 0.001 \\ \text { Zhao W, 2020 } & 0.011 & 0.002 & 0.072 & -4.497 & 0.000 \\ \text { Feng Z, 2020 } & 0.043 & 0.019 & 0.091 & -7.462 & 0.000 \\ \text { Yuan M, 2020 } & 0.018 & 0.001 & 0.230 & -2.808 & 0.005 \\ \text { Wu J, 2020 } & 0.039 & 0.013 & 0.115 & -5.418 & 0.000 \\ \text { Chung M, 2020 } & 0.026 & 0.002 & 0.310 & -2.519 & 0.012 \\ \text { Song F, 2020 } & 0.060 & 0.019 & 0.170 & -4.621 & 0.000 \\ \text { Bernheim A, 2020 } & 0.005 & 0.000 & 0.079 & -3.697 & 0.000 \\ \text { Xu Z, 2020 } & 0.176 & 0.058 & 0.427 & -2.421 & 0.015 \\ \text { Li K, 2020 } & 0.084 & 0.041 & 0.166 & -6.038 & 0.000 \\ \text { Liu J, 2020 } & 0.008 & 0.001 & 0.121 & -3.353 & 0.001 \\ & 0.047 & 0.030 & 0.075 & -12.127 & 0.000\end{array}$

\section{Event rate and $95 \% \mathrm{Cl}$}

0.000
0.000
0.000
0.000
0.000
0.000
0.001
0.000
0.000
0.000
0.000
0.000
0.001
0.000

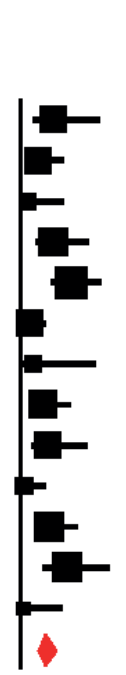

lelative weight

8.32

8.54

3.50

10.05

12.96

8.64

3.45

9.42

8.40

3.52

10.73

10.49

1.97

Heterogeneitv: $\mathrm{I}^{2}=\mathbf{3 2 . 0 7 \%}, \mathrm{P}=\mathbf{0 . 1 2 6}$

0.00

0.50

1.00

Event rate and $95 \% \mathrm{Cl}$

Meta Analysis

Figure 4. Cont. 
Viruses are a common cause of respiratory tract infection. Imaging findings of viral pneumonia are varied, and may overlap with other infectious and inflammatory lung diseases. Viruses in the same viral family have a similar pathogenesis, so chest CT scan may help identify distinct patterns and features in immunocompromised patients [56]. Meta-analysis of initial data suggests that chest CT scan findings for 2019-nCoV have many features simi-

A

Study name

Evr

Xu Y H, 2020

$\mathrm{Sh} \mathrm{H}, 2020$

Li Y, 2020

Chung M, 2020

Bernheim A, 2020

Li K, 2020

Liu J, 2020

0.345
0.584

0.190

0.745

0.778

0.564

0.747

0.345
Statistics for each study

$\begin{array}{ccc}\begin{array}{c}\text { Event } \\ \text { rate }\end{array} & \text { limit } & \text { limit }\end{array}$

0.732

0.577

0.165

0.845

Z-Value

0.609

0.217

2.846

$-16.590$

0.535

0.846

3.338

0.462

0.914

2.210

0.660

1.234

0.643

0.829

4.288

0.234

0.475

$-2.323$

0.336

0.795

0.653
Event rate and $95 \% \mathrm{Cl}$

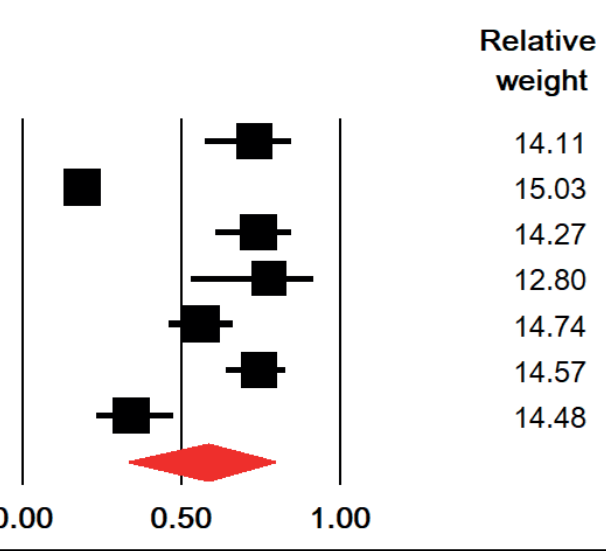

Meta Analysis

B

Study name

$\begin{array}{lccrrr} & \begin{array}{c}\text { Event } \\ \text { rate }\end{array} & \begin{array}{c}\text { Lower } \\ \text { limit }\end{array} & \begin{array}{c}\text { Upper } \\ \text { limit }\end{array} & \text { Z-Value } & \text { p-Value } \\ \text { Xu Y H, 2020 } & 0.537 & 0.385 & 0.681 & 0.468 & 0.640 \\ \text { Sh H, 2020 } & 0.102 & 0.084 & 0.125 & -19.176 & 0.000 \\ \text { Li Y, 2020 } & 0.765 & 0.630 & 0.861 & 3.570 & 0.000 \\ \text { Chung M, 2020 } & 0.667 & 0.429 & 0.842 & 1.386 & 0.166 \\ \text { Bernheim A, 2020 } & 0.532 & 0.431 & 0.630 & 0.618 & 0.536 \\ \text { Li K, 2020 } & 0.735 & 0.630 & 0.819 & 4.101 & 0.000 \\ \text { Liu J, 2020 } & 0.276 & 0.176 & 0.404 & -3.285 & 0.001 \\ & 0.497 & 0.230 & 0.766 & -0.017 & 0.987\end{array}$

0.987
Event rate and $95 \% \mathrm{Cl}$

Relative weight

14.26

14.74

14.20

13.47

14.56

14.46

14.32

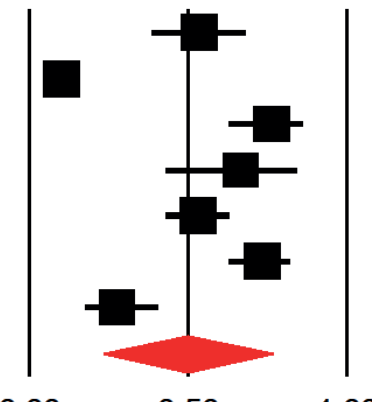

Heterogeneity: $\mathrm{I}^{2}=\mathbf{9 7 . 7 4} \%, \mathrm{P}<\mathbf{0 . 0 0 1}$

1.00

C

Study name

$\begin{array}{lrrrrr} & \begin{array}{c}\text { Event } \\ \text { rate }\end{array} & \begin{array}{c}\text { Lower } \\ \text { limit }\end{array} & \begin{array}{c}\text { Upper } \\ \text { limit }\end{array} & \text { Z-Value } & \text { p-Value } \\ \text { Xu Y H, 2020 } & 0.951 & 0.825 & 0.988 & 4.097 & 0.000 \\ \text { Sh H, 2020 } & 0.265 & 0.236 & 0.296 & -13.118 & 0.000 \\ \text { Li Y, 2020 } & 0.980 & 0.874 & 0.997 & 3.873 & 0.000 \\ \text { Chung M, 2020 } & 0.889 & 0.648 & 0.972 & 2.773 & 0.006 \\ \text { Bernheim A, 2020 } & 0.840 & 0.752 & 0.901 & 5.899 & 0.000 \\ \text { Li K, 2020 } & 0.940 & 0.863 & 0.975 & 5.955 & 0.000 \\ \text { Liu J, 2020 } & 0.776 & 0.651 & 0.865 & 3.944 & 0.000 \\ & 0.865 & 0.577 & 0.968 & 2.354 & 0.019\end{array}$

0.019
Event rate and $95 \% \mathrm{Cl}$

Relative

weight

13.68

15.45

12.34

13.57

15.17

14.69

15.10

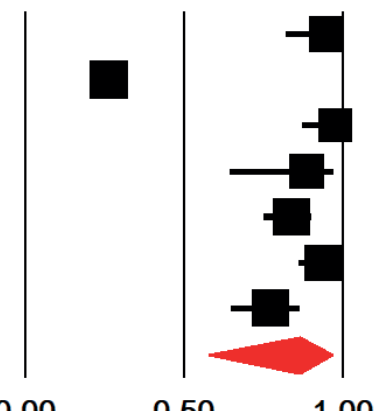

Heterogeneity: $\mathrm{I}^{2}=\mathbf{9 7 . 4 6 \%}, \mathrm{P}<\mathbf{0 . 0 0 1}$

.00

Figure 5. Meta-analysis of right upper lobe (A), right middle lobe (B), right lower lobe (C), left upper lobe (D), and left lower lobe (E) involvement in chest computed tomography scan of COVID-19 pneumonia 
Study name

\begin{tabular}{|c|c|c|c|c|c|c|}
\hline & $\begin{array}{l}\text { Event } \\
\text { rate }\end{array}$ & $\begin{array}{c}\text { Lower } \\
\text { limit }\end{array}$ & $\begin{array}{c}\text { Upper } \\
\text { limit }\end{array}$ & Z-Value & p-Value & $\begin{array}{r}\text { Relative } \\
\text { weight }\end{array}$ \\
\hline Xu Y H, 2020 & 0.805 & 0.656 & 0.899 & 3.596 & 0.000 & 14.02 \\
\hline Sh H, 2020 & 0.204 & 0.178 & 0.232 & -15.996 & 0.000 & 14.98 \\
\hline Li Y, 2020 & 0.784 & 0.651 & 0.876 & 3.792 & 0.000 & 14.27 \\
\hline Chung M, 2020 & 0.778 & 0.535 & 0.914 & 2.210 & 0.027 & 13.08 \\
\hline Bernheim A, 2020 & 0.617 & 0.515 & 0.709 & 2.248 & 0.025 & 14.72 \\
\hline Li K, 2020 & 0.855 & 0.762 & 0.916 & 5.696 & 0.000 & 14.38 \\
\hline \multirow[t]{2}{*}{ Liu J, 2020} & 0.379 & 0.264 & 0.509 & -1.820 & 0.069 & 14.54 \\
\hline & 0.645 & 0.373 & 0.847 & 1.048 & 0.295 & \\
\hline Heterogeneity: $\mathrm{I}^{2}=97.3$ & $0, P<0.001$ & & & & 0.00 & \\
\hline
\end{tabular}

Statistics for each study

Analysis

E

\section{Study name}

Study name

Xu Y H, 2020

Sh H, 2020

Li Y, 2020

Chung M, 2020

Bernheim A, 2020

Li K, 2020

Liu J, 2020
Statistics for each study

Event
rate

Lower limit

\section{Upper}

limit Z-Value
0.739

0.878

0.238

0.941

0.778

0.809

0.964

0.707

0.810
0.739
0.210

0.833

0.535

0.716

0.894

0.578

0.505
0.948

0.268

0.981

0.914

0.876

0.988

0.809

0.947

4.136
-14.443
4.659
2.210
5.495
5.583
3.052
1.987

p-Value

0.000
0.000
0.000
0.027
0.000
0.000
0.002
0.047

Event rate and $95 \% \mathrm{Cl}$

Relative

weight

14.17

15.05

13.71

13.82

14.79

13.74

14.73

Heterogeneity: $\mathrm{I}^{2}=\mathbf{9 7 . 6 1 \%}, \mathrm{P}<\mathbf{0 . 0 0 1}$ Meta Analysis

Figure 5. Cont.

lar to other viruses such as the middle east respiratory syndrome (MERS-CoV) and severe acute respiratory syndrome (SARS-CoV) [57].

In the present study, most pulmonary lesions include bilateral lung involvement with multiple lung lobes (predominantly right lower lobe and left lower lobe), with dominant distribution in the peripheral portion of the lungs. Studies have shown that influenza pneumonia tends to affect the lower lobes [56,58]. Wang et al. also showed that H7N9 pneumonia has a predominant distribution in the right lower lobe [58]. Both H1N1 and SARS pneumonia are more peripherally distributed $[59,60]$, whereas no lobe infection is found in H5N1 influenza [61]. However, lung involvement with peripheral predominance has also been observed in SARS and MERS. Similarly, previous coronavirus pneumonias have a similar pattern. The dominant peripheral distribution for COVID-19 was shown in our study. Such a distribution is obvious at first glance. This feature of chest CT scan is caused by alveolar injury and pulmonary interstitial edema. We also observed some chest CT scan features of COVID-19 that differ from chest CT scan features of SARS and MERS. Unifocal involvement is more common than multifocal involvement in patients with SARS and patients with MERS [62,63]. However, contrary to what is seen in the chest CT scan of patients with COVID-19, multi-lobe involvement was more common than single-lobe involvement in the present meta-analysis. Thus, more than two lobes are likely to be involved in this disease. To the best of our knowledge, these findings have not been reported in the literature related to SARS and MERS.

Our results showed that the most common findings of imaging were pure GGO, GGO with mixed consolidation or reticular pattern, interlobular septal thickening, and consolidation. Reticular and nodular pattern were relatively small, which may be explained in the first stage of the disease. In H7N9 pneumonia, most cases showed consolidation [62]. Each of the chest CT scan patterns in our patients is nonspecific and may overlap with other microorganism infections such as H7N9 pneumonia, 
H1N1 virus infection, SARS, MERS, and avian influenza A (H5N1) [60-63].

GGO, consolidation, and interlobular septal thickening are the most common chest $\mathrm{CT}$ scan findings of $\mathrm{H} 1 \mathrm{~N} 1$ influenza pneumonia, too [59]. Based on the present meta-analysis, pure GGO is a common finding in about $65 \%$ of cases, and GGO with interlobular septal thickening/ consolidation in $49 \%$ of COVID-19 pneumonia patients. Thus, these features of chest CT scan can be seen in most patients. This finding, along with the dominant distribution in the peripheral part of the lungs, is not common in other viral pneumonias $[8,18,27,29,60,61]$.

In addition, we found that all features of chest CT scan that exist in the initial chest CT scan of patients with COVID-19, such as GGO and consolidation, and other chest CT scan features such as vascular enlargement, interlobular septal thickening, and air bronchogram sign, are also present in the chest CT scan of SARS and MERS. The low incidence of pleural effusion and lymphadenopathy noted in our data was also a feature of chest CT scan in previous studies about SARS [57]. This may be due to the inherent anatomical features of the lower lobe bronchus. The right lower lobe bronchus is tighter than other bronchi of the lung, and the angle between the right lower lobe and the long axis of the trachea is smaller, so it is more viral at early stages. Most likely, it attacks the bronchial branches of the lower lobe and causes infection.

Interestingly enough, we found that most patients have vascular enlargement lesions $(61.4 \%)$ that may be caused by an acute inflammatory response. However, vascular changes are not similar to changes in malignant lesions such as lung adenocarcinoma that cause vascular dilatation or irregularity and vascular convergence, which may be due to chronic progression and tumor infiltration [17,64].

Angiotensin II converting enzyme is a key molecule involved in the development and progression of acute lung failure. COVID-19 induces direct lung injury by involving angiotensin converting enzyme, which contributes to the progression of alveolar injury [65]. This may explain the pathological mechanism of GGO and consolidation as well as rapid changes in chest CT scan findings. Our results support the observed process, according to which bilateral GGOs or mixed GGOs in chest CT scan should prompt the radiologist to recommend COVID-19 as a possible diagnosis $[66,67]$.

\section{Conclusions}

In summary, this work is a meta-analysis on preliminary studies of chest CT scan findings about COVID-19, aimed at introducing the common imaging manifestations of the disease. Radiologists play an important role in rapid iden- tification and early detection of new cases, which can be useful not only for the patient but also for public health surveillance systems. It is important to recognize the fact that the appearance of CT findings about COVID-19 has some similarities to other viral diseases, especially those in the same viral family (SARS and MERS). Future studies are recommended to determine how CT scans of COVID-19 patients change after treatment.

Limitations of this study include the following: 1 . All studies were performed in China, and the severity of chest CT scan manifestations might be affected by ethnic factors; 2 . Most patients were hospitalized patients and patients with milder symptoms or those who were not hospitalized, which may cause bias in the results; 3 . In most preliminary studies, chest CT scan findings were not separately reviewed according to patients admitted to the ICU or the isolation ward; 4 . Follow-up for chest CT scan during treatment until discharge was not included in our study; 5. Our results are based on CT findings during admission, but patients might have experienced symptoms before admission (because chest CT scan findings are influenced by the clinical course of the disease), and during this time the patient might have received antiviral or antibacterial drugs, or steroid therapy, and this might have affected the chest CT scan findings; 6 . Since all studies were performed in 2020 in China with the same diagnostic method, we could not discover the cause of heterogeneity.

In conclusion, the most important outcomes in chest CT scan of patients with COVID-19 pneumonia were bilateral lung involvement, GGO or mixed (GGO pulse consolidation or reticular) patterns, thickened interlobular septa, vascular enlargement, air bronchogram sign, peripheral distribution, left and right lower lobes involvement, and one or five lobes involvement.

Our study showed that chest CT scan has high sensitivity in diagnosis of COVID-19, and may therefore serve as a standard method for diagnosis of COVID-19. Rapid detection may lead to early control of the transmission. By diagnosing viral pneumonia on CT scan, infected or suspected patients can be isolated and treated in a timely manner to optimize patient management, especially for hospitals or communities without RT-PCR test kits. However, chest CT scan is still limited in terms of identifying specific viruses. It is important that radiologists recognize whether chest CT scan findings for COVID-19 overlap with chest CT scan findings for diseases caused by different virus families, such as adenovirus.

\section{Conflict of interest}

The authors report no conflict of interest. 


\section{References}

1. WHO. Coronavirus disease (COVID-19) outbreak 2020. Available from: https://www.who.int/westernpacific/emergencies/covid-19.

2. Li Q, Guan X, Wu P, et al. Early transmission dynamics in Wuhan, China, of novel coronavirus-infected pneumonia. N Engl J Med 2020; 382: 1199-1207.

3. Chen N, Zhou M, Dong X, et al. Epidemiological and clinical characteristics of 99 cases of 2019 novel coronavirus pneumonia in $\mathrm{Wu}$ han, China: a descriptive study. Lancet 2020; 395: 507-513.

4. Jones KE, Patel NG, Levy MA, et al. Global trends in emerging infectious diseases. Nature 2008; 451: 990-993.

5. Woolhouse ME, Haydon DT, Antia R. Emerging pathogens: the epidemiology and evolution of species jumps. Trends Ecol Evol 2005; 20: 238-244.

6. Alizon S, Hurford A, Mideo N, et al. Virulence evolution and the trade-off hypothesis: history, current state of affairs and the future. J Evol Biol 2009; 22: 245-259.

7. Pan Y, Guan H. Imaging changes in patients with 2019-nCov. Eur Radiol 2020; 30: 3612-3613.

8. Huang C, Wang Y, Li X, et al. Clinical features of patients infected with 2019 novel coronavirus in Wuhan, China. Lancet 2020; 395: 497-506.

9. Chen G, Wu D, Guo W, et al. Clinical and immunologic features in severe and moderate forms of Coronavirus Disease 2019. J Clin Invest 2020; 130: 2620-2629.

10. Yang W, Cao Q, Qin L, et al. Clinical characteristics and imaging manifestations of the 2019 novel coronavirus disease (COVID-19): a multi-center study in Wenzhou city, Zhejiang, China. J Infect 2020; 80: 388-393.

11. Xu Y-H, Dong J-H, An W-M, et al. Clinical and computed tomographic imaging features of novel coronavirus pneumonia caused by SARS-CoV-2. J Infect 2020; 80: 394-400.

12. Shi $H$, Han $X$, Jiang $N$, et al. Radiological findings from 81 patients with COVID-19 pneumonia in Wuhan, China: a descriptive study. Lancet Infect Dis 2020; 20: 425-434.

13. Wang D, Hu B, Hu C, et al. Clinical characteristics of 138 hospitalized patients with 2019 novel coronavirus-infected pneumonia in Wuhan, China. Jama 2020; 323: 1061-1069.

14. Pan $\mathrm{Y}$, Guan $\mathrm{H}$, Zhou S, et al. Initial CT findings and temporal changes in patients with the novel coronavirus pneumonia (2019$\mathrm{nCoV})$ : a study of 63 patients in Wuhan, China. Eur Radiol 2020; 30: 3306-3309.

15. Xu X-W, Wu X-X, Jiang X-G, et al. Clinical findings in a group of patients infected with the 2019 novel coronavirus (SARS-Cov-2) outside of Wuhan, China: retrospective case series. BMJ 2020; 368 : m792.

16. Li Y, Xia L. Coronavirus Disease 2019 (COVID-19): role of chest CT in diagnosis and management. AJR Am J Roentgenol 2020; 214: 1-7.

17. Zhou S, Wang Y, Zhu T, et al. CT features of coronavirus disease 2019 (COVID-19) pneumonia in 62 patients in Wuhan, China. AJR Am J Roentgenol 2020; 214: 1287-1294.

18. Zhao W, Zhong Z, Xie X, et al. Relation between chest CT findings and clinical conditions of coronavirus disease (COVID-19) pneumonia: a multicenter study. AJR Am J Roentgenol 2020; 214 1072-1077.
19. Guan W-j, Ni Z-y, Hu Y, et al. Clinical characteristics of 2019 novel coronavirus infection in China. N Engl J Med 2020; 382: 1708-1720.

20. Ai J-W, Zhang H-C, Xu T, et al. Optimizing diagnostic strategy for novel coronavirus pneumonia, a multi-center study in Eastern China. Available from: https://www.medrxiv.org/content/10.1101/202 0.02.13.20022673v1.

21. Feng Z, Yu Q, Yao S, et al. Early prediction of disease progression in 2019 novel coronavirus pneumonia patients outside wuhan with CT and clinical characteristics. Available from: https://www.medrxiv. org/content/10.1101/2020.02.19.20025296v1.

22. Yuan M, Yin W, Tao Z, et al. Association of radiologic findings with mortality of patients infected with 2019 novel coronavirus in $\mathrm{Wu}$ han, China. Plus One 2020; 15: e0230548.

23. Qian G-Q, Yang N-B, Ding F, et al. Epidemiologic and clinical characteristics of 91 hospitalized patients with COVID-19 in Zhejiang, China: a retrospective, multi-centre case series. QJM 2020; 113 : 474-481.

24. Cao W. Clinical features and laboratory inspection of novel coronavirus pneumonia (COVID-19) in Xiangyang, Hubei. Available from: https://www.medrxiv.org/content/10.1101/2020.02.23.20026963v1.

25. Liang Y, Liang J, Zhou Q, et al. Prevalence and clinical features of 2019 novel coronavirus disease (COVID-19) in the Fever Clinic of a teaching hospital in Beijing: a single-center, retrospective study. Available from: https://www.medrxiv.org/content/10.1101/2020.0 $2.25 .20027763 \mathrm{v} 2$.

26. Huang $\mathrm{Y}$, Zhou H, Yang R, et al. Clinical characteristics of 36 non-survivors with COVID-19 in Wuhan, China. Available from: https://www.medrxiv.org/content/10.1101/2020.02.27.20029009v2.

27. Wu J, Wu X, Zeng W, et al. Chest CT findings in patients with corona virus disease 2019 and its relationship with clinical features. Invest Radiol 2020; 55: 257-261.

28. Kui L, Fang Y-Y, Deng Y, et al. Clinical characteristics of novel coronavirus cases in tertiary hospitals in Hubei Province. Chin Med J 2020; 133: 1025-1031.

29. Chung M, Bernheim A, Mei X, et al. CT imaging features of 2019 novel coronavirus (2019-nCoV). Radiology 2020; 295: 202-207.

30. Song F, Shi N, Shan F, et al. Emerging 2019 novel coronavirus (2019nCoV) pneumonia. Radiology 2020; 295: 210-217.

31. Bernheim A, Mei X, Huang M, et al. Chest CT findings in coronavirus disease-19 (COVID-19): relationship to duration of infection. Radiology 2020; 295.

32. Xu Z, Wu W, Jin Y, et al. Key points of clinical and CT imaging features of 2019 novel coronavirus (2019-ncov) imported pneumonia based on 21 cases analysis. Available from: https://www.medrxiv. org/content/10.1101/2020.03.03.20030775v1.

33. Li K, Wu J, Wu F, et al. The clinical and chest $\mathrm{CT}$ features associated with severe and critical COVID-19 pneumonia. Invest Radiol 2020; 55: 327-331.

34. Zhang J-J, Dong X, Cao Y-Y, et al. Clinical characteristics of 140 patients infected with SARS-CoV-2 in Wuhan, China. Allergy 2020; 75: $1730-1741$.

35. Liu W, Wang F, Li G, et al. Analysis of 2019-nCoV infection and clinical manifestations of outpatients: an epidemiological study from the fever clinic in Wuhan, China. 2020. 
36. Hu Z, Song C, Xu C, et al. Clinical characteristics of 24 asymptomatic infections with COVID-19 screened among close contacts in Nanjing, China. Sci China Life Sci 2020; 63: 706-711.

37. Fu H, Li H, Tang X, et al. Analysis on the clinical characteristics of 36 cases of novel coronavirus pneumonia in Kunming. Available from: https://www.medrxiv.org/content/10.1101/2020.02.28.20029173v1.

38. Li J, Li S, Cai Y, et al. Epidemiological and clinical characteristics of 17 hospitalized patients with 2019 novel coronavirus infections outside Wuhan, China. Available from: https://www.medrxiv.org/ content/10.1101/2020.02.11.20022053v1.

39. Cai Q, Huang D, Ou P, et al. COVID-19 in a designated infectious diseases hospital outside Hubei Province, China. Allergy 2020; 75: 1742-1752.

40. Jian-ya G. Clinical characteristics of 51 patients discharged from hospital with COVID-19 in Chongqing, China. Available from: https://www.medrxiv.org/content/10.1101/2020.02.20.20025536v1.

41. Cui P, Chen Z, Wang T, et al. Clinical features and sexual transmission potential of SARS-CoV-2 infected female patients: a descriptive study in Wuhan, China. Available from: https://www.medrxiv.org/ content/10.1101/2020.02.26.20028225v2.

42. Cao M, Zhang D, Wang Y, et al. Clinical features of patients infected with the 2019 novel coronavirus (COVID-19) in Shanghai, China. Available from: https://www.medrxiv.org/content/10.1101/2020.03 $.04 .20030395 \mathrm{v} 1$.

43. Xu Y, Li Y-r, Zeng Q, et al. Clinical characteristics of SARS-CoV-2 pneumonia compared to controls in Chinese Han population. Available from: https://www.medrxiv.org/content/10.1101/2020.03.08.2 $0031658 v 1$.

44. Chen J, Fan H, Zhang L, et al. Retrospective analysis of clinical features in 101 death cases with COVID-19. Available from: https:// www.medrxiv.org/content/10.1101/2020.03.09.20033068v2.

45. Liu J, Ouyang L, Guo P, et al. Epidemiological, clinical characteristics and outcome of medical staff infected with COVID-19 in Wuhan, China: a retrospective case series analysis. Available from: https://www.medrxiv.org/content/10.1101/2020.03.09.20033118v1.

46. Lei Y, Lan Y, Lu J, et al. Clinical features of imported cases of coronavirus disease 2019 in Tibetan patients in the Plateau area. Available from: https://www.medrxiv.org/content/10.1101/2020.03.09.2 $0033126 v 1$.

47. Sayehmiri K, Tavan H, Sayehmire F, et al. Prevalence of epilepsy in Iran using meta-analysis and systematic review. J Adv Med Biomed Res 2015; 23: 112-121.

48. Sayehmiri K, Tavan H. Systematic review and meta-analysis methods prevalence of peptic ulcer in IRAN. Govaresh 2016; 20: 250-258.

49. Stroup DF, Berlin JA, Morton SC, et al. Meta-analysis of observational studies in epidemiology: a proposal for reporting. Jama 2000; 283: 2008-2012.

50. Moher D, Shamseer L, Clarke M, et al. Preferred reporting items for systematic review and meta-analysis protocols (PRISMA-P) 2015 statement. Syst Rev 2015; 4: 1.

51. Peterson J, Welch V, Losos M, et al. The Newcastle-Ottawa scale (NOS) for assessing the quality of nonrandomised studies in meta-analyses. Available from: http://www.ohri.ca/programs/clinical_epidemiology/oxford.asp.

52. Higgins JP, Green S. Cochrane handbook for systematic reviews of interventions. John Wiley \& Sons, New Jersey 2011.
53. Higgins JP, Thompson SG, Deeks JJ, et al. Measuring inconsistency in meta-analyses. BMJ 2003; 327: 557-560.

54. Xie X, Zhong Z, Zhao W, et al. Chest CT for typical 2019-nCoV pneumonia: relationship to negative RT-PCR testing. Radiology 2020; 296: E41-E45.

55. National Health Commission of the People's Republic of China. China's Hubei reports jump in new cases of COVID-19 after diagnosis criteria revision. Available from: http://en.nhc.gov.cn/202002/13/c_76515.htm.

56. Koo HJ, Lim S, Choe J, et al. Radiographic and CT features of viral pneumonia. Radiographics 2018; 38: 719-739.

57. Ooi GC, Khong PL, Müller NL, et al. Severe acute respiratory syndrome: temporal lung changes at thin-section CT in 30 patients. Radiology 2004; 230: 836-844.

58. Wang Q, Zhang Z, Shi Y, et al. Emerging H7N9 influenza A (novel reassortant avian-origin) pneumonia: radiologic findings. Radiology 2013; 268: 882-889.

59. Yuan Y, Tao XF, Shi YX, et al. Initial HRCT findings of novel influenza A (H1N1) infection. Influenza and other respiratory viruses. Influenza Other Respir Viruses 2012; 6: e114-e119.

60. Wong K-t, Antonio GE, Hui DS, et al. Severe acute respiratory syndrome: thin-section computed tomography features, temporal changes, and clinicoradiologic correlation during the convalescent period. J Comput Assist Tomogr 2004; 28: 790-795.

61. Qureshi NR, Hien TT, Farrar J, et al. The radiologic manifestations of H5N1 avian influenza. Journal of thoracic imaging. J Thorac Imaging; 2006; 21: 259-264.

62. Paul NS, Roberts H, Butany J, et al. Radiologic pattern of disease in patients with severe acute respiratory syndrome: the Toronto experience. Radiographics 2004; 24: 553-563.

63. Das KM, Lee EY, Langer RD, et al. Middle east respiratory syndrome coronavirus: what does a radiologist need to know? AJR Am J Roentgenol 2016; 206: 1193-1201.

64. Xu X, Chen P, Wang J, et al. Evolution of the novel coronavirus from the ongoing Wuhan outbreak and modeling of its spike protein for risk of human transmission. Sci China Life Sci 2020; 63: 457-460.

65. Zhou P, Yang X-L, Wang X-G, et al. A pneumonia outbreak associated with a new coronavirus of probable bat origin. Nature 2020; 579: 270-273.

66. Müller NL, Ooi GC, Khong PL, et al. Severe acute respiratory syndrome: radiographic and CT findings. AJR Am J Roentgenol 2003; 181: 3-8.

67. Müller NL, Ooi GC, Khong PL, et al. High-resolution CT findings of severe acute respiratory syndrome at presentation and after admission. AJR Am J Roentgenol 2004; 182: 39-44. 